Document downloaded from:

http://hdl.handle.net/10251/105524

This paper must be cited as:

Capilla Romá, MT.; Talavera Usano, CF.; Ginestar Peiro, D.; Verdú Martín, GJ. (2017). A study of the radiative transfer equation using a spherical harmonics-nodal collocation method. Journal of Quantitative Spectroscopy and Radiative Transfer. 189:25-36. doi:10.1016/j.jqsrt.2016.11.008

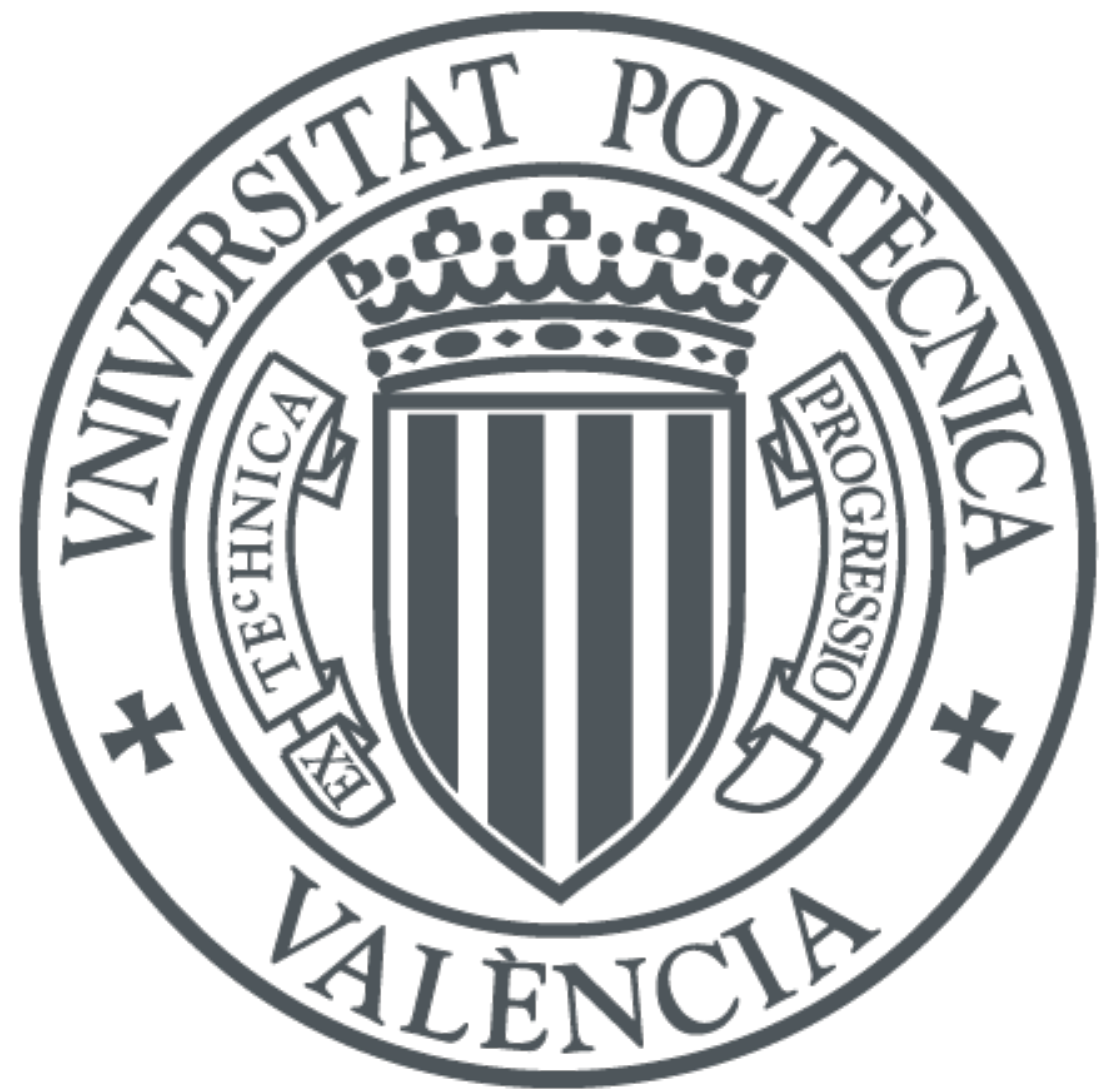

The final publication is available at

http://dx.doi.org/10.1016/j.jqsrt.2016.11.008

Copyright Elsevier

Additional Information 


\title{
A study of the radiative transfer equation using a spherical harmonics-nodal collocation method
}

\author{
M. T. Capilla ${ }^{\mathrm{a}}$, C. F. Talavera ${ }^{\mathrm{a}}$, D. Ginestar ${ }^{\mathrm{a}}$, G. Verdúb ${ }^{\mathrm{b}, *}$ \\ ${ }^{a}$ Departamento de Matemática Aplicada, Universitat Politècnica de València, Camino de \\ Vera 14, E-46022 Valencia, Spain \\ ${ }^{b}$ Departamento de Ingeniería Química y Nuclear, Universitat Politècnica de València, \\ Camino de Vera 14, E-46022 Valencia, Spain
}

\begin{abstract}
Optical tomography has found many medical applications that need to know how the photons interact with the different tissues. The majority of the photon transport simulations are done using the diffusion approximation, but this approximation has a limited validity when optical properties of the different tissues present large gradients, when structures near the photons source are studied or when anisotropic scattering has to be taken into account. As an alternative to the diffusion model, the $P_{L}$ equations for the radiative transfer problem are studied. These equations are discretized in a rectangular mesh using a nodal collocation method. The performance of this model is studied by solving different $1 \mathrm{D}$ and $2 \mathrm{D}$ benchmark problems of light propagation in tissue having media with isotropic and anisotropic scattering.
\end{abstract}

Keywords: radiative transfer equation, light propagation in tissue, multidimensional $P_{L}$ equations, spherical harmonics, nodal collocation method PACS: 02.60.Lj, 05.60.Cd, 87.57.-s, 87.90.+y

\section{Introduction}

Optical tomography, also referred to as diffuse optical tomography, has made considerable advances in recent years $[1,2]$. This field is concerned with the use of visible and near-infrared light for diagnosis and treatment of biological tissues. Examples include optical monitoring of blood oxygenation, detection of cerebral hemorrhages, functional imaging of brain activity, diagnosis of Alzheimer's disease, breast cancer, etc. [3].

The use of optical techniques for medical imaging is an attractive alternative to other methods using ionizing radiation [4]. Optical imaging of the breast has undergone an important growth [5] as an imaging technique for the detection and

\footnotetext{
* Corresponding author.

Email addresses: tcapilla@mat.upv.es (M. T. Capilla), talavera@mat.upv.es

(C. F. Talavera), dginesta@mat.upv.es (D. Ginestar), gverdu@iqn.upv.es (G. Verdú)
}

Preprint submitted to Journal of Quantitative Spectroscopy and Radiative TransferNovember 7, 2016 
diagnosis of cancer. Optical techniques have also been applied to the detection and diagnosis of brain cancer, Alzheimer and other pathologies $[6,7,8,9]$. These methods are different from other imaging techniques based on ionizing radiation because the light at the intensities used for diagnostic is not carcinogenic. A further motivation for using optical techniques based on photons is that these are sensitive to information related to metabolic processes and blood flow $[3,8]$, identifying different types of soft tissues. However, optical photons created in the near-infrared light spectrum suffer from scattering by heterogeneous bodies giving significant amount of blurring.

Having an efficient model to describe photon transport in tissue is fundamental in the medical application of optical radiation. Modeling of light propagation in tissue is largely done through the use of the diffusion approximation to the radiative transfer equation. But the diffusion approximation is only valid in optically thick media (the system must be large compared to the photon mean free path), far from any boundary layer, and when the absorption coefficient is small compared to the scattering coefficient. Since most biological tissues obey this condition, the diffusion approximation is often a good approximation and is frequently solved using finite elements techniques [10]. However, this model is not applicable to non-scattering void-like layers where the scattering and absorption are very low [11], and to regions where optical properties change a lot from the surrounding region [12]. Also, structures near to boundary source [13], clear regions, and anisotropic scattering cannot be fully taken into account with the diffusion approximation [14].

Providing solutions to the radiative transfer equation (RTE) remains a challenging task in the fields of tissue optics and radiological sciences. Limits of diffusion approximation have been investigated and compared with other methods by several researchers. Thus, in [9] the authors use a time-independent, discrete ordinates $\left(S_{N}\right)$, finite difference transport code called DANTSYS that was developed by Alcouffe and co-workers and present the comparison of finite-difference transport and diffusion calculations for photon migration in homogeneous and heterogeneous tissues. To overcome known problems of the discrete ordinates method as the ray effect, the angular discretization of the RTE can be performed using spherical harmonics series. If the series is truncated at the $L$ th moment, the $P_{L}$ approximation is obtained [12]. To try to overcome the complexity of the full $P_{L}$ theory, an approximation based on simplified spherical harmonics, $S P_{L}$, has been successfully applied to $2 \mathrm{D}$ electron-photon problems [15], and to tissue optics [16]. Although the $S P_{L}$ equations are fewer equations to solve than the full $P_{L}$ equations, the $S P_{L}$ solutions do not converge to exact transport solutions. On the other hand, the diffusive spherical harmonics $P_{L}$ equations have several advantages regarding the $S_{N}$ or $S P_{L}$ equations. First, the diffusive $P_{L}$ equations approximates the RTE equations by a set of coupled diffusion-like equations, which can be solved with standard diffusion solvers. Also, they do not suffer from ray effects, as the $S_{N}$ method does. Yet, $P_{L}$ equations can lead to highly oscillatory behavior and even negative particle concentrations $[17,18]$. In the work of $[6,19]$ the authors developed a finite element spherical harmonics radiation transport model that is applied to this type of problems. All these 
issues strongly suggest that the diffusive $P_{L}$ spherical-harmonics equations can be applied to optical tomography, hence our interest in revisiting this field of medical imaging research.

In this paper, a spherical harmonics-nodal collocation method for the solution of the diffusive (second order) $P_{L}$ equations is proposed. The angular $P_{L}$ approximation is computed for arbitrary odd $L$. The spatial discretization is based on a nodal collocation method and the use of Legendre polynomials series on a coarse spatial mesh. The method has a rapid convergence with the number of Legendre polynomials, and the size of the discretized problem is smaller than the one obtained with other techniques as the finite difference method, when the same accuracy is required.

The present work is organized as follows: In Section 2, we introduce the radiative transfer equation for light propagation in tissue, and we review the multi-dimensional spherical harmonics $P_{L}$ equations for arbitrary angular order $L$, that will be formulated as a vector-valued diffusive second order differential equation. Surface source and vacuum boundary conditions are approximated using Marshak's conditions and will be computed for arbitrary order $L$. The spatial discretization is then carried out using a nodal collocation method. In Section 3, several photon propagation problems are analyzed and used to validate the numerical accuracy of the $P_{L}$ method described in Section 2. We examine the transport of photons through $1 \mathrm{D}$ and $2 \mathrm{D}$ homogeneous media, and also through $2 \mathrm{D}$ heterogeneous media with void-like regions and channels. In both types of media, cases with isotropic and anisotropic scattering are studied, considering in the last cases the Henyey-Greenstein and simplified approximated Mie phase functions. Finally the main conclusions of the study are summarized in Section 4.

\section{The radiative transfer equation}

The propagation of light in scattering media can be described by the radiative transfer equation (RTE), that is, the linearized one-speed, steady-state form of the Boltzmann transport equation [20]:

$$
\vec{\Omega} \vec{\nabla} \Phi(\vec{r}, \vec{\Omega})+\mu_{t}(\vec{r}) \Phi(\vec{r}, \vec{\Omega})=\int d \vec{\Omega}^{\prime} \mu_{s}\left(\vec{r} ; \vec{\Omega}^{\prime} \rightarrow \vec{\Omega}\right) \Phi\left(\vec{r}, \vec{\Omega}^{\prime}\right)+S(\vec{r}, \vec{\Omega}),
$$

where $\Phi(\vec{r}, \vec{\Omega})$ is the photon angular flux at the spatial point $\vec{r}=\left(x_{1}, x_{2}, x_{3}\right)$ and unit direction-of-flight $\vec{\Omega}=(\cos \varphi \sin \theta, \sin \varphi \sin \theta, \cos \theta), 0<\varphi<2 \pi$, $0<\theta<\pi ; \mu_{t}$ is the total macroscopic cross-section (attenuation coefficient), $\mu_{s}$ is the scattering cross-section from direction $\vec{\Omega}^{\prime}$ to direction $\vec{\Omega}$ and finally $S(\vec{r}, \vec{\Omega})$ is the internal fixed source term.

In the spherical harmonics method the angular dependence of the photon flux $\Phi(\vec{r}, \vec{\Omega})$ and the source term $S(\vec{r}, \vec{\Omega})$ are expanded in terms of the (com-

plex) spherical harmonics $Y_{l}^{m}(\vec{\Omega})=\sqrt{\frac{(2 l+1)}{4 \pi} \frac{(l-m) !}{(l+m) !}} P_{l}^{m}(\cos \theta) e^{i m \varphi}[21]$ (where $P_{l}^{m}(\cos \theta)$ are the associated Legendre polynomials), that form a complete set 
of orthonormal functions, that is, they satisfy the orthonormality property $\int d \vec{\Omega} Y_{m}^{l}{ }^{*} Y_{m^{\prime}}^{l^{\prime}}=\delta_{l l^{\prime}} \delta_{m m^{\prime}}$, where $\delta_{i j}$ is the Kronecker delta and $d \vec{\Omega}=\sin \theta d \varphi d \theta$, $0<\varphi<2 \pi, 0<\theta<\pi$. Thus,

$$
\begin{aligned}
& \Phi(\vec{r}, \vec{\Omega})=\sum_{l=0}^{\infty} \sum_{m=-l}^{+l} \phi_{l m}(\vec{r}) Y_{l}^{m}(\vec{\Omega}), \\
& S(\vec{r}, \vec{\Omega})=\sum_{l=0}^{\infty} \sum_{m=-l}^{+l} s_{l m}(\vec{r}) Y_{l}^{m}(\vec{\Omega}),
\end{aligned}
$$

where $\phi_{l m}(\vec{r})$ and $s_{l m}(\vec{r})$ are the (spherical harmonics) moments. Complex spherical harmonics will provide a more concise theoretical description of the method. The isotropic photon flux is then $\phi(\vec{r})=\frac{1}{4 \pi} \int d \vec{\Omega} \Phi(\vec{r}, \vec{\Omega})=(4 \pi)^{-1 / 2} \phi_{00}(\vec{r})$.

It will also be assumed that scattering depends only on the relative angle between the incident and the scattered photons, $\vec{\Omega} \vec{\Omega}^{\prime}$, and that the scattering cross-section may be expanded as the following series of Legendre polynomials

$$
\mu_{s}\left(\vec{r}, \vec{\Omega} \vec{\Omega}^{\prime}\right)=\sum_{l=0}^{\infty} \frac{2 l+1}{4 \pi} \mu_{s, l}(\vec{r}) P_{l}\left(\vec{\Omega} \vec{\Omega}^{\prime}\right) .
$$

In the process of light scattering in tissues the scattering cross-section is factorized as $[22]$

$$
\mu_{s}\left(\vec{r}, \vec{\Omega} \vec{\Omega}^{\prime}\right)=\mu_{s}(\vec{r}) p\left(\vec{\Omega} \vec{\Omega}^{\prime}\right),
$$

where the phase function $p$ is given by theoretical models and provides a convenient description of anisotropic scattering in terms of a unique parameter, the anisotropy factor $\mathrm{g}$. Due to its simplicity, the most widely adopted is the Henyey-Greenstein (H-G) function [23]:

$$
p_{\mathrm{HG}}\left(\vec{\Omega} \vec{\Omega}^{\prime}\right)=\frac{1-g^{2}}{4 \pi\left(1+g^{2}-2 g \vec{\Omega} \vec{\Omega}^{\prime}\right)^{3 / 2}}=\sum_{l=0}^{\infty} \frac{2 l+1}{4 \pi} g^{l} P_{l}\left(\vec{\Omega} \vec{\Omega}^{\prime}\right)
$$

( $g=0$ corresponds to isotropic scattering). From (3) it is straightforward that the expansion coefficients are

$$
\mu_{s, l}^{\mathrm{HG}}(\vec{r})=\mu_{s}(\vec{r}) g^{l}, \quad l=0,1, \ldots
$$

This model is inefficient in the forward peaked range $(g>0.8)$ due to the high number of Legendre expansion coefficients required [6]. So recently a new scattering phase function, the simplified approximate Mie (SAM), has been proposed [24] that requires less coefficients:

$$
p_{\mathrm{SAM}}\left(\vec{\Omega} \vec{\Omega}^{\prime}\right)=K_{S}\left(1+\vec{\Omega} \vec{\Omega}^{\prime}\right)^{n_{p}},
$$

where $n_{p}=\frac{2 g}{1-g}$ is the anisotropic index and $K_{S}=\frac{1}{2 \pi} \frac{n_{p}+1}{2^{n} p+1}$ is the normalization factor. Now, the expansion coefficients in (3) are given by

$$
\mu_{s, l}^{\mathrm{SAM}}(\vec{r})=\mu_{s}(\vec{r}) \frac{n_{p}+1}{2^{n_{p}+1}} \int_{-1}^{1}(1+\omega)^{n_{p}} P_{l}(\omega) d \omega, \quad l=0,1, \ldots
$$


Expansions (2) and (3) and the orthogonality properties of $Y_{l}^{m}$ are then used into (1). Let us consider the first term in (1), that accounts for photons removed by leakage in direction $\vec{\Omega}$. If $\vec{\Omega} \vec{\nabla} \Phi(\vec{r}, \vec{\Omega})=\sum_{l^{\prime}=0}^{\infty} \sum_{m^{\prime}=-l^{\prime}}^{+l^{\prime}} A_{l^{\prime} m^{\prime}}(\vec{r}) Y_{l^{\prime}}^{m^{\prime}}(\vec{\Omega})$ then (for simplicity, we omit the arguments of $Y_{l}^{m}$ and $\Phi$ )

$$
\begin{aligned}
A_{l^{\prime} m^{\prime}}=\int d \vec{\Omega} Y_{l^{\prime}}^{m^{\prime *}}(\vec{\Omega} \vec{\nabla} \Phi) & \\
=\sum_{\substack{l=0 \\
-l \leq m \leq+l}}^{\infty}\left[\int d \vec{\Omega} Y_{l^{\prime}}^{m^{\prime *}} \cos \varphi \sin \theta Y_{l}^{m} \frac{\partial \phi_{l m}}{\partial x_{1}}+\right. & \int d \vec{\Omega} Y_{l^{\prime}}^{m^{\prime *}} \sin \varphi \sin \theta Y_{l}^{m} \frac{\partial \phi_{l m}}{\partial x_{2}} \\
& \left.+\int d \vec{\Omega} Y_{l^{\prime}}^{m^{\prime *}} \cos \theta Y_{l}^{m} \frac{\partial \phi_{l m}}{\partial x_{3}}\right]
\end{aligned}
$$

If we have into account the following formulas

$$
\begin{aligned}
\int d \vec{\Omega} Y_{l^{\prime}}^{m^{\prime *}} \sin \theta e^{-i \varphi} Y_{l}^{m} & =\left(-C_{1}(l, m) \delta_{l-1, l^{\prime}}+C_{2}\left(l^{\prime}, m^{\prime}\right) \delta_{l^{\prime}-1, l}\right) \delta_{m^{\prime}+1, m}, \\
\int d \vec{\Omega} Y_{l^{\prime}}^{m^{\prime *}} \sin \theta e^{i \varphi} Y_{l}^{m} & =\left(-C_{1}\left(l^{\prime}, m^{\prime}\right) \delta_{l^{\prime}-1, l}+C_{2}(l, m) \delta_{l-1, l^{\prime}}\right) \delta_{m+1, m^{\prime}}, \\
\int d \vec{\Omega} Y_{l^{\prime}}^{m^{\prime *}} \cos \theta Y_{l}^{m} & =\left(C_{3}(l, m) \delta_{l-1, l^{\prime}}+C_{3}\left(l^{\prime}, m^{\prime}\right) \delta_{l^{\prime}-1, l}\right) \delta_{m^{\prime}, m},
\end{aligned}
$$

where

$$
\begin{aligned}
& C_{1}(l, m)=\left(\frac{(l+m)(l+m-1)}{(2 l+1)(2 l-1)}\right)^{1 / 2}, \\
& C_{2}(l, m)=C_{1}(l,-m), \\
& C_{3}(l, m)=\left(\frac{(l+m)(l-m)}{(2 l+1)(2 l-1)}\right)^{1 / 2},
\end{aligned}
$$

it follows that

$$
\begin{aligned}
& A_{l^{\prime} m^{\prime}}=\sum_{\substack{l=0 \\
-l \leq m \leq+l}}^{\infty}\left\{\frac { 1 } { 2 } \left[\left(-C_{1}(l, m) \delta_{l-1, l^{\prime}}+C_{2}\left(l^{\prime}, m^{\prime}\right) \delta_{l^{\prime}-1, l}\right) \delta_{m^{\prime}+1, m}\right.\right. \\
&\left.+\left(-C_{1}\left(l^{\prime}, m^{\prime}\right) \delta_{l^{\prime}-1, l}+C_{2}(l, m) \delta_{l-1, l^{\prime}}\right) \delta_{m+1, m^{\prime}}\right] \frac{\partial \phi_{l m}}{\partial x_{1}} \\
&+ \frac{1}{2 i}\left[\left(-C_{1}(l, m) \delta_{l-1, l^{\prime}}+C_{2}\left(l^{\prime}, m^{\prime}\right) \delta_{l^{\prime}-1, l}\right) \delta_{m^{\prime}+1, m}\right. \\
&+\left.\left(-C_{1}\left(l^{\prime}, m^{\prime}\right) \delta_{l^{\prime}-1, l}+C_{2}(l, m) \delta_{l-1, l^{\prime}}\right) \delta_{m+1, m^{\prime}}\right] \frac{\partial \phi_{l m}}{\partial x_{2}} \\
&+\left.\left(C_{3}(l, m) \delta_{l-1, l^{\prime}}+C_{3}\left(l^{\prime}, m^{\prime}\right) \delta_{l^{\prime}-1, l}\right) \delta_{m^{\prime}, m} \frac{\partial \phi_{l m}}{\partial x_{3}}\right\} .
\end{aligned}
$$

Let us now consider the scattering source term in the equation (1). Knowing that the Legendre polynomials of a scalar product of unit vectors can be expanded as $P_{l}\left(\vec{\Omega} \vec{\Omega}^{\prime}\right)=\frac{4 \pi}{2 l+1} \sum_{m=-l}^{l} Y_{l}^{m}(\vec{\Omega}) Y_{l}^{m *}\left(\vec{\Omega}^{\prime}\right)$, and using the assumption 
(3), we get

$$
\begin{aligned}
& \int d \vec{\Omega}^{\prime} \sum_{l=0}^{\infty} \frac{2 l+1}{4 \pi} \mu_{s, l}(\vec{r}) P_{l}\left(\vec{\Omega} \vec{\Omega}^{\prime}\right) \Phi\left(\vec{r}, \vec{\Omega}^{\prime}\right) \\
= & \int d \vec{\Omega}^{\prime} \sum_{l=0}^{\infty} \sum_{m=-l}^{l} \mu_{s, l}(\vec{r}) Y_{l}^{m}(\vec{\Omega}) Y_{l}^{m *}\left(\vec{\Omega}^{\prime}\right) \Phi\left(\vec{r}, \vec{\Omega}^{\prime}\right)=\sum_{\substack{l=0 \\
l \leq m \leq+l}}^{\infty} \mu_{s, l} Y_{l}^{m}(\vec{\Omega}) \phi_{l m}(\vec{r}) .
\end{aligned}
$$

From these expressions it is straightforward to obtain the following (infinite) set of (complex) equations for the spherical harmonics moments $\phi_{l m}$ :

$$
\begin{array}{r}
\frac{1}{2}\left(-C_{1}(l+1, m+1) \frac{\partial \phi_{l+1, m+1}}{\partial x_{1}}+C_{2}(l, m) \frac{\partial \phi_{l-1, m+1}}{\partial x_{1}}\right. \\
\left.\quad-C_{1}(l, m) \frac{\partial \phi_{l-1, m-1}}{\partial x_{1}}+C_{2}(l+1, m-1) \frac{\partial \phi_{l+1, m-1}}{\partial x_{1}}\right) \\
+\frac{1}{2 i}\left(-C_{1}(l+1, m+1) \frac{\partial \phi_{l+1, m+1}}{\partial x_{2}}+C_{2}(l, m) \frac{\partial \phi_{l-1, m+1}}{\partial x_{2}}\right. \\
\left.\quad-C_{1}(l, m) \frac{\partial \phi_{l-1, m-1}}{\partial x_{2}}+C_{2}(l+1, m-1) \frac{\partial \phi_{l+1, m-1}}{\partial x_{2}}\right) \\
+C_{3}(l+1, m) \frac{\partial \phi_{l+1, m}}{\partial x_{3}}+C_{3}(l, m) \frac{\partial \phi_{l-1, m}}{\partial x_{3}}+\mu_{t} \phi_{l m} \\
=\mu_{s, l} \phi_{l m}+s_{l m}, \quad l=0,1, \ldots, \quad m=-l, \ldots,+l .
\end{array}
$$

It is understood that terms involving moments $\phi_{l m}$ with invalid indices $l$ and $m$ are zero. To obtain a finite approximation, the series in expansions (2) and (3) are truncated at some finite order $l=L$ and the resulting equations (7) are known as the $P_{L}$ equations. In the following, we will only consider $L$ to be an odd integer.

We observe that the radiative transfer equation (1) is a real equation and, as we are interested (for physical reasons) on real solutions, then $\Phi=\Phi^{*}$, that is, $\phi_{l m}{ }^{*}=(-1)^{m} \phi_{l,-m}$ and not all complex moments are independent so there are only $2 l+1$ real independent moments for each $l>0$, that is, $\left\{\phi_{l 0}, \operatorname{Re} \phi_{l m}, \operatorname{Im} \phi_{l m}, m=1, \ldots, l\right\}$. We will now obtain the real form of $P_{L}$ equations (7). Taking real and imaginary part in (7) and defining the real moments

$$
\begin{aligned}
\xi_{l m} & =\operatorname{Re} \phi_{l m}=\frac{1}{2}\left(\phi_{l m}+(-1)^{m} \phi_{l,-m}\right), \\
\eta_{l m} & =\operatorname{Im} \phi_{l m}=\frac{1}{2 i}\left(\phi_{l m}-(-1)^{m} \phi_{l,-m}\right),
\end{aligned}
$$


we obtain the corresponding $2 l+1$ real equations:

$$
\begin{aligned}
& \frac{1}{2}\left(-C_{1}(l+1, m+1) \frac{\partial \xi_{l+1, m+1}}{\partial x_{1}}+C_{2}(l, m) \frac{\partial \xi_{l-1, m+1}}{\partial x_{1}}\right. \\
& \left.\quad-C_{1}(l, m) \frac{\partial \xi_{l-1, m-1}}{\partial x_{1}}+C_{2}(l+1, m-1) \frac{\partial \xi_{l+1, m-1}}{\partial x_{1}}\right) \\
& +\frac{1}{2}\left(-C_{1}(l+1, m+1) \frac{\partial \eta_{l+1, m+1}}{\partial x_{2}}+C_{2}(l, m) \frac{\partial \eta_{l-1, m+1}}{\partial x_{2}}\right. \\
& \left.\quad-C_{1}(l, m) \frac{\partial \eta_{l-1, m-1}}{\partial x_{2}}+C_{2}(l+1, m-1) \frac{\partial \eta_{l+1, m-1}}{\partial x_{2}}\right) \\
& +C_{3}(l+1, m) \frac{\partial \xi_{l+1, m}}{\partial x_{3}}+C_{3}(l, m) \frac{\partial \xi_{l-1, m}}{\partial x_{3}}+\mu_{t} \xi_{l m} \\
& =\mu_{s, l} \xi_{l m}+\operatorname{Re} s_{l m}, \quad m=0,1, \ldots, l,
\end{aligned}
$$

and

$$
\begin{aligned}
& \frac{1}{2}\left(-C_{1}(l+1, m+1) \frac{\partial \eta_{l+1, m+1}}{\partial x_{1}}+C_{2}(l, m) \frac{\partial \eta_{l-1, m+1}}{\partial x_{1}}\right. \\
& \left.-C_{1}(l, m) \frac{\partial \eta_{l-1, m-1}}{\partial x_{1}}+C_{2}(l+1, m-1) \frac{\partial \eta_{l+1, m-1}}{\partial x_{1}}\right) \\
& -\frac{1}{2}\left(-C_{1}(l+1, m+1) \frac{\partial \xi_{l+1, m+1}}{\partial x_{2}}+C_{2}(l, m) \frac{\partial \xi_{l-1, m+1}}{\partial x_{2}}\right. \\
& \left.\quad-C_{1}(l, m) \frac{\partial \xi_{l-1, m-1}}{\partial x_{2}}+C_{2}(l+1, m-1) \frac{\partial \xi_{l+1, m-1}}{\partial x_{2}}\right) \\
& +C_{3}(l+1, m) \frac{\partial \eta_{l+1, m}}{\partial x_{3}}+C_{3}(l, m) \frac{\partial \eta_{l-1, m}}{\partial x_{3}}+\mu_{t} \eta_{l m} \\
& =\mu_{s, l} \eta_{l m}+\operatorname{Im} s_{l m}, \quad m=1, \ldots, l,
\end{aligned}
$$

From the index structure of the equations (9) and (10), it is convenient to gather even $l$ moments into vectors

$$
X=\left(\xi_{l, m \geq 0}, \eta_{l, m>0}\right)_{l=\text { even }}, \quad \mathcal{S}=\left(\operatorname{Re} s_{l, m \geq 0}, \operatorname{Im} s_{l, m>0}\right)_{l=\text { even }},
$$

with $n_{e}=L(L+1) / 2$ components, and odd $l$ moments into vectors

$$
\bar{X}=\left(\xi_{l, m \geq 0}, \eta_{l, m>0}\right)_{l=\text { odd }}, \quad \overline{\mathcal{S}}=\left(\operatorname{Re} s_{l, m \geq 0}, \operatorname{Im} s_{l, m>0}\right)_{l=\text { odd }},
$$

with $n_{o}=(L+1)(L+2) / 2$ components (for example, if $L=1$ then $X=\left(\xi_{00}\right)$ and $\left.\bar{X}=\left(\xi_{10}, \xi_{11}, \eta_{11}\right)^{T}\right)$. Then (9) and (10) can be rewritten as

$$
\begin{aligned}
& \sum_{j=1}^{3} M_{j} \frac{\partial \bar{X}}{\partial x_{j}}+\operatorname{diag}\left(\mu_{t}-\mu_{s l}\right)_{l=\text { even }}=\mathcal{S} \\
& \sum_{j=1}^{3} \bar{M}_{j} \frac{\partial X}{\partial x_{j}}+\operatorname{diag}\left(\mu_{t}-\mu_{s l}\right)_{l=\text { odd }} \bar{X}=\overline{\mathcal{S}}
\end{aligned}
$$


where $M_{j}$ and $\bar{M}_{j}$ are rectangular matrices of dimensions $n_{e} \times n_{o}$ and $n_{o} \times n_{e}$ respectively, defined from the coefficients of (9) and (10). Eq. (14) relates $\bar{X}$ with derivatives of $X$ so it corresponds to a generalization of "Fick's law":

$$
\bar{X}=-D \sum_{j=1}^{3} \bar{M}_{j} \frac{\partial X}{\partial x_{j}}+D \overline{\mathcal{S}},
$$

where $D=\operatorname{diag}\left(\mu_{t}-\mu_{s l}\right)_{l=\text { odd }}^{-1}$ is a square matrix. Replacing (15) into (13) we obtain the "diffusive form of $P_{L}$ equations":

$$
-\sum_{i, j=1}^{3} \frac{\partial}{\partial x_{i}}\left(M_{i} D \bar{M}_{j} \frac{\partial X}{\partial x_{j}}\right)+\mu_{a} X=\mathcal{S}_{\mathrm{eff}},
$$

where $\mu_{a}=\operatorname{diag}\left(\mu_{t}-\mu_{s l}\right)_{l=\text { even }}$ is the absorption coefficient and the "effective source term" is $\mathcal{S}_{\text {eff }}=\mathcal{S}-\sum_{j=1}^{3} \frac{\partial}{\partial x_{j}}\left(M_{j} D \overline{\mathcal{S}}\right)$. The square "effective diffusion matrices" $M_{i} D \bar{M}_{j}$ generalize the diffusion coefficient $1 /\left(3\left(\mu_{t}-\mu_{s 1}\right)\right)$ of $P_{1}$ equation to $P_{L}$ equations for $L>1$. Notice that the equation (16) will encounter difficulties when dealing with problems that involve void regions, where matrix $D$ is (near) singular.

Finally, (16) corresponds to 3D geometry. Lower dimensional geometries are obtained by imposing restrictions to the angular flux. The XY (2D) geometry is obtained considering that the angular flux does not depend on the third coordinate, $\Phi=\Phi\left(x_{1}, x_{2}, \vec{\Omega}\right)$, so $\frac{\partial \Phi}{\partial x_{3}}=0$, and also must satisfy the symmetry relation $\Phi(\theta)=\Phi(\pi-\theta)$, so the moments $\phi_{l m}=0$ if $l+m$ is odd. The planar (1D) geometry is obtained by imposing that the photon flux $\Phi=\Phi\left(x_{3}, \theta\right)$ so the only non-zero moments are $\phi_{l, m=0}=\xi_{l 0}$ and they are also real.

\subsection{Boundary conditions}

When an external surface source is located at the boundary of the region described by (1), the angular photon flux is specified by $T(r, \vec{\Omega})$ for every incoming direction,

$$
\Phi(\vec{r}, \vec{\Omega})=T(r, \vec{\Omega}), \quad \text { for all } \vec{\Omega} \vec{n} \leq 0,
$$

where $\vec{n}$ is the outwardly directed unitary normal vector to the external surface. Vacuum boundary conditions, in particular, correspond to $T=0$. This condition can be approximated by setting Marshak's conditions [25]

$$
\int_{\vec{\Omega} \vec{n} \leq 0} d \vec{\Omega} Y_{l}^{m *}(\vec{\Omega})(\Phi(\vec{r}, \vec{\Omega})-T(\vec{r}, \vec{\Omega}))=0,
$$

for $l=1,3,5, \ldots, L$ (odd) and $m=0,1, \ldots, l$ (the conditions with negative $m$ index are redundant because the photon flux $\Phi$ is a real function). Notice that (18) is complex so there are $2 l+1$ real conditions for each odd index $l$.

We will only consider regions with prismatic geometry; we can then use the symmetry $Y_{l}^{m}(-\vec{\Omega})=(-1)^{l} Y_{l}^{m}(\vec{\Omega})$ obtaining that, for $l+l^{\prime}$ even,

$$
\int_{\vec{\Omega} \vec{n} \leq 0} d \vec{\Omega} Y_{l}^{m *}(\vec{\Omega}) Y_{l^{\prime}}^{m^{\prime}}(\vec{\Omega})=\frac{1}{2} \int_{\vec{\Omega}} d \vec{\Omega} Y_{l}^{m *}(\vec{\Omega}) Y_{l^{\prime}}^{m^{\prime}}(\vec{\Omega})=\frac{1}{2} \delta_{l l^{\prime}} \delta_{m m^{\prime}} .
$$


Inserting the expansion given by the equation (2) for $\Phi$ and also for the external source,

$$
T(\vec{r}, \vec{\Omega})=\sum_{l=0}^{\infty} \sum_{m=-l}^{+l} T_{l m}(\vec{r}) Y_{l}^{m}(\vec{\Omega})
$$

truncated up to a finite odd order $L$, into Marshak's conditions (18) and using (19), it results into the conditions

$$
\frac{1}{2}\left(\phi_{l m}-T_{l m}\right)+\sum_{\substack{l^{\prime} \text { even } \\-l^{\prime} \leq m^{\prime} \leq l^{\prime}}}^{L-1}\left(\int_{\vec{\Omega} \vec{n} \leq 0} d \vec{\Omega} Y_{l}^{m *}(\vec{\Omega}) Y_{l^{\prime}}^{m^{\prime}}(\vec{\Omega})\right)\left(\phi_{l^{\prime} m^{\prime}}-F_{l^{\prime} m^{\prime}}\right)=0
$$

for $l=1,3,5, \ldots, L$ and $m=0,1, \ldots, l$. Taking real and imaginary part in (20), Marshak's conditions can be written as

$$
(\bar{X}-\bar{T})+N^{V}(X-T)=0,
$$

that is,

$$
\bar{X}+N^{V} X=\bar{T}+N^{V} T,
$$

where real vectors $X$ and $\bar{X}$ were defined in (11) and (12), also

$$
T=\left(\operatorname{Re} T_{l, m \geq 0}, \operatorname{Im} T_{l, m>0}\right)_{l=\text { even }}, \quad \bar{T}=\left(\operatorname{Re} T_{l, m \geq 0}, \operatorname{Im} T_{l, m>0}\right)_{l=\text { odd }},
$$

and $N^{V}$ is a real rectangular matrix (of dimensions $n_{o} \times n_{e}$ ) whose numerical values depend on the geometry of the boundary surface, that is, the spatial axis normal to the boundary surface.

\subsection{The nodal collocation method for an isotropic source}

Since $P_{L}$ equations (16) have a diffusive form, their spatial discretization can be done using a nodal collocation method, previously used for the neutron diffusion equation $[26,27]$ and generalized for eigenvalue problems in multidimensional rectangular geometries [28, 29]. For simplicity, in this work we will only apply the method when the internal source $S(\vec{r}, \vec{\Omega})$ in (1) is isotropic. This implies that $\overline{\mathcal{S}}=0$ and no source term appears in Fick's law (15). This situation was studied in [29] so we will only briefly describe the method for 3D geometry.

Given an structured rectilinear mesh with vertex coordinates $\left\{x_{1, i_{1}}, x_{2, i_{2}}, x_{3, i_{3}}\right\}$, where $i_{j}=0,1, \ldots, m_{j}(j=1,2,3)$ are vertex indices, we only consider a domain that can be divided into $N\left(\leq m_{1} m_{2} m_{3}\right)$ adjacent rectangular prisms, or nodes, of the form $N^{e}=\left[x_{1, i_{1}}, x_{1, i_{1}+1}\right] \times\left[x_{2, i_{2}}, x_{2, i_{2}+1}\right] \times\left[x_{3, i_{3}}, x_{3, i_{3}+1}\right]$, being $e=1, \ldots, N$ the index that enumerates the nodes, once an appropriate node ordering has been chosen, see Fig. 1.

For a generic node $N^{e}$ the following change of variables

$$
u_{j}=\frac{1}{\Delta x_{j}^{e}}\left(x_{j}-\frac{1}{2}\left(x_{j, i_{j}}+x_{j, i_{j}+1}\right)\right), \quad j=1,2,3,
$$




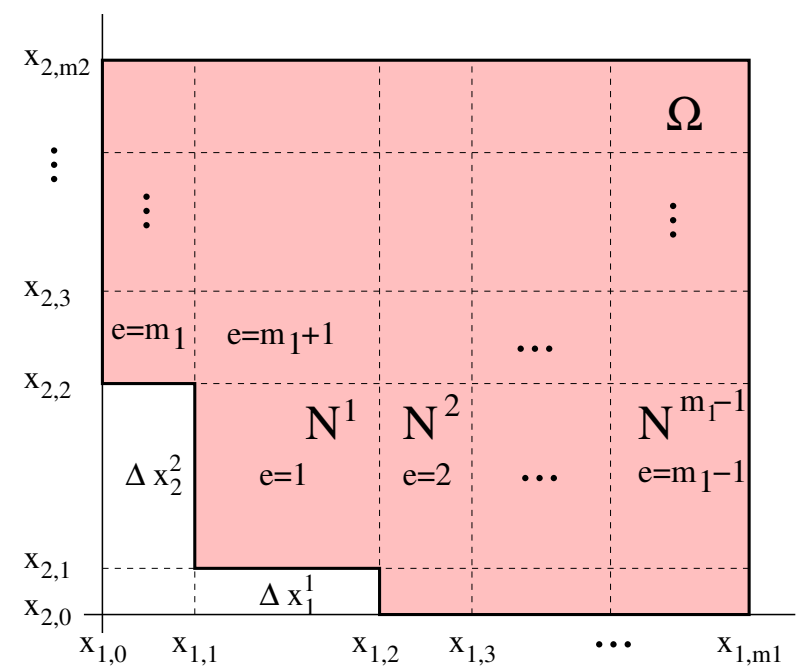

Figure 1: Sample rectilinear mesh covering the domain for 2D geometry. A natural ordering for spatial nodes $N^{e}$ has been chosen.

where $\Delta x_{j}^{e}=x_{j, i_{j}+1}-x_{j, i_{j}}$, transforms the node $N^{e}$ into the cubic node of volume one $N_{u}^{e}=\left[-\frac{1}{2},+\frac{1}{2}\right]^{3}$.

The nodal collocation method assumes that on each node the cross-sections and the internal source term in (1) are constant. For each node $N^{e}$, the $P_{L}$ equations (16) are transformed by means of the change of variables (23). Furthermore, if $X^{e}\left(u_{1}, u_{2}, u_{3}\right)$ denotes the previously defined vector of $l$ even moments that appears in (16) for node $N^{e}$, it is assumed that spatial dependence of vector $X^{e}$ can be expanded in terms of (orthonormal) Legendre polynomials $\mathcal{P}_{k}(u)[28]$ up to a certain finite order $M$,

$$
X^{e}\left(u_{1}, u_{2}, u_{3}\right)=\sum_{k_{1}, k_{2}, k_{3}=0}^{M} x_{k_{1} k_{2} k_{3}}^{e} \mathcal{P}_{k_{1}}\left(u_{1}\right) \mathcal{P}_{k_{2}}\left(u_{2}\right) \mathcal{P}_{k_{2}}\left(u_{3}\right)
$$

where $u_{j} \in\left[-\frac{1}{2},+\frac{1}{2}\right], j=1,2,3$, and the Legendre coefficients $x_{k_{1} k_{2} k_{3}}^{e}$ are the unknowns to be determined. Notice that polynomial expansion of the source term $\mathcal{S}_{\text {eff }}^{e}$ at node $N^{e}$ reduces to the constant term. The series (24) is then inserted into (16) and equations for $x_{k_{1} k_{2} k_{3}}^{e}$ are derived using the orthonormality properties of $\mathcal{P}_{k}(u)$.

Double derivative terms in (16) will involve coupling with neighboring nodes. When node $N^{e}$ is an interior node, adjacent nodes are related imposing continuity of the photon angular flux $\Phi(\vec{r}, \vec{\Omega})$ (or, equivalently, of all moments $X^{e}$ and $\left.\bar{X}^{e}\right)$ at the interface between nodes. In the case that the node $N^{e}$ is adjacent to a boundary, then Marshak's boundary conditions (22) are used. See [28, 29] for further details. 
Finally, once an appropriate ordering of the indices is chosen, the previous procedure approximates the equations (16) by an algebraic problem that can be casted in the form

$$
\mathcal{A} V=\mathcal{S},
$$

where $V$ is a real vector of components $\left(\xi_{l, m \geq 0 ; k_{1} k_{2} k_{3}}^{e}, \eta_{l, m>0 ; k_{1} k_{2} k_{3}}^{e}\right), \mathcal{S}$ is the independent term associated with the source term and the external surface source and $\mathcal{A}$ is a matrix of dimension

$$
N \times N_{\text {Leg }} \times n_{e}=N \times M^{d} \times \frac{L(L+1)}{2},
$$

where $N$ is the number of nodes; $N_{\text {Leg }}$ is the number of Legendre moments, being $M$ the order in Legendre series (24) and $d$ the spatial dimension; and finally $n_{e}$ is the number of components of vector $X$ in (11), being $L$ the order of the $P_{L}$ approximation. Problem (25) is a system of linear equations that is large and sparse. The linear system is then iteratively solved using the bi-conjugate gradient stabilized method BICGSTAB, with an incomplete LU factorization, ILUT, as preconditioner, from the FORTRAN library SPARSKIT [30].

In previous works $[28,29]$ we investigated the convergence of the nodal collocation method, with different number of discretization nodes and different order $M$ of Legendre polynomials used in the expansion (24). We observed that the convergence is largely improved increasing the degree of the polynomials considered (for $M=1$, there is a slow spatial convergence). Also, we observed that, from the computational point of view, it is more favorable increasing the polynomial order $M$ than increasing the number of discretization nodes [29].

\section{Numerical results}

In this Section, we present some numerical results in order to evaluate the performance and numerical accuracy of the nodal collocation method. The method has been already tested with neutron transport problems that are driven by internal sources [31] and also with eigenvalue problems [29]. The formulation described above has been implemented in the multi-group radiation transport code SHNC (Spherical Harmonics-Nodal Collocation), written in FORTRAN 90, which solves the external fixed source problem for an arbitrary $P_{L}$ approximation for odd $L$, and we show its application as a light propagation model for biological homogeneous and heterogeneous tissues by choosing appropriate numerical examples.

We present results for a one-dimensional problem, comparing the numerical $P_{L}$ results to the analytical solution. Also we present several two-dimensional cases where we compare the $P_{L}$ photon fluxes to $S_{N}$ results obtained from the code DANTSYS [32], taken as reference. In some cases our solutions are also compared to solutions from the literature. Computation times vary from several seconds to several minutes, depending on the size of the spatial mesh and the order of the $P_{L}$ approximation, on a workstation with AMD Phenom II 1055T processor and $16 \mathrm{~Gb}$ of memory, using only one processor for each computation. 
Since the primary purpose of this work is to verify the capability of the SHNC code to solve the photon propagation problems with accuracy with respect to reference results, providing details of the numerical $P_{L}$ approximations, the computational efficiency in solving the $P_{L}$ equations was not the priority of this study.

\subsection{One-dimensional homogeneous medium}

To validate our code, we first analyze a test example consisting of a simple homogeneous one-dimensional region of finite length, where analytical solution exists.

We obtain the 1D transport equation for plane symmetry when the medium is transversely infinite (in the $x_{1} x_{2}$ plane) with cross sections and source variation only in the $x_{3}$ direction. For this case, the radiative transfer equation (1) becomes

$$
\omega \frac{d \Phi}{d z}(z, \omega)+\mu_{t} \Phi(z, \omega)=0, \quad 0<z<l,
$$

where we have defined $z=x_{3}, \omega=\cos \theta \in[-1,1]$, then the photon flux $\Phi=$ $\Phi(z, \omega)$; the scattering and absorption coefficients considered for this region are $\mu_{s}=0$ and $\mu_{a} \neq 0$, far away from a diffusive $\left(P_{1}\right)$ regime. Finally, we set an external source $T$ at $z=0$ and vacuum boundary condition at the other side:

$$
\begin{aligned}
& \Phi(0, \omega)=T(0, \omega), \quad \text { if } 0<\omega \leq+1, \\
& \Phi(l, \omega)=0, \quad \text { if } \quad-1 \leq \omega<0 .
\end{aligned}
$$

If $\omega \neq 0$ is kept fixed, the general solution of the first order ordinary differential equation $(27)$ is $\Phi(z, \omega)=C(\omega) e^{-\frac{\mu_{t}}{\omega} z}$, where $C(\omega)$ is a function only of $\omega$. If we impose the boundary conditions $(28)$ we get

$$
\Phi(z, \omega)=\left\{\begin{array}{cl}
T(0, \omega) e^{-\frac{\mu_{t}}{\omega} z} & \text { if } 0<\omega \leq+1 \\
0 & \text { if }-1 \leq \omega<0 .
\end{array}\right.
$$

Then the isotropic photon flux is

$$
\phi(z)=\frac{1}{2} \int_{-1}^{+1} d \omega \Phi(z, \omega)=\frac{1}{2} \int_{0}^{+1} d \omega T(0, \omega) e^{-\frac{\mu_{t}}{\omega} z} .
$$

For the numerical calculations a slab of length $10 \mathrm{~mm}$ is considered, with an isotropic external source $T(0, \omega)=1$ photons $/ \mathrm{mm}$ located at $z=0 \mathrm{~mm}$ and vacuum boundary condition applied at the right boundary, as shown in Fig. 2. The absorption coefficient considered is $\mu_{a}=0.1 \mathrm{~mm}^{-1}$.

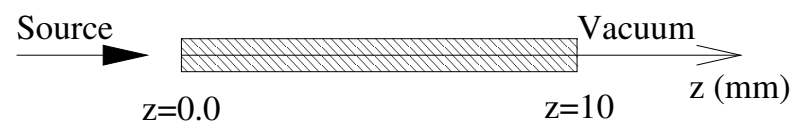

Figure 2: Geometry of the one-dimensional slab problem. 
We have performed the numerical $P_{L}$ solutions for the photon fluxes with the SHNC code. We have used for the calculations a mesh consisting of 10 nodes with size $1 \mathrm{~mm}$ and the order of the Legendre polynomials expansion considered in (24) is $M=4$.

Fig. 3 shows the photon flux as function of the distance $z$ from the external source, calculated with the $P_{L}$ approximations for $L=1,3,7,9$, together with the analytical solution (29), obtained by numerical integration using Quadpack [33]. The figure also shows the $S_{24}$ solution obtained with the discrete-ordinates code ONEDANT [32], with 1000 mesh points.

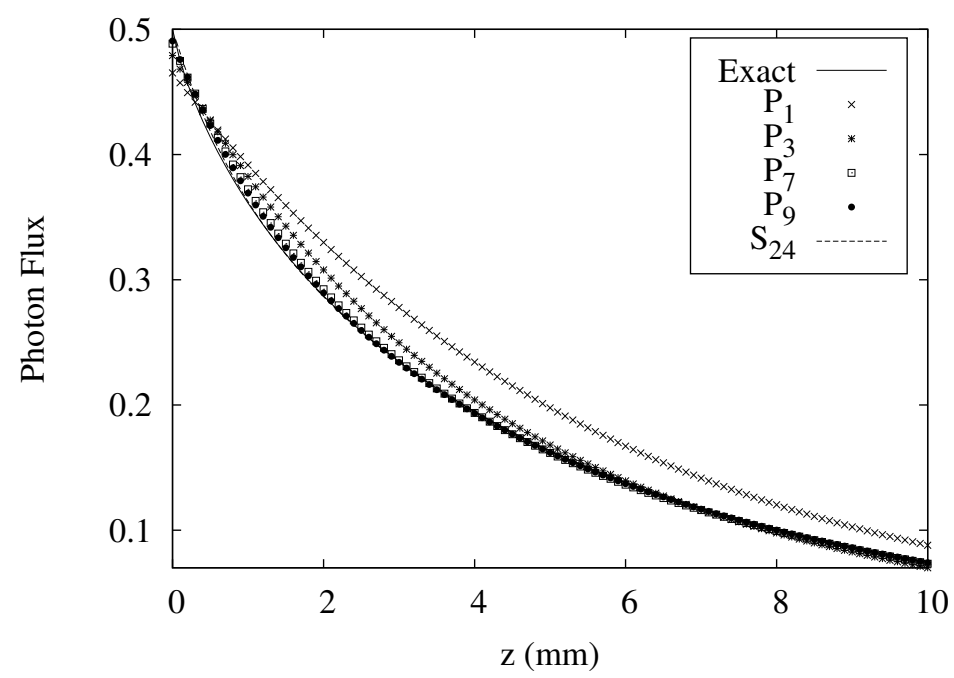

Figure 3: Photon fluxes for the homogeneous one-dimensional problem.

As can be seen from this figure, the $P_{1}$ approximation, as expected, fails to describe the light propagation correctly, whereas higher order $P_{L}$ approximations $\left(P_{7}, P_{9}\right)$ show accurate results in comparison to the exact solution.

\subsection{Two-dimensional homogeneous media}

A second example to study the application of the nodal collocation method in homogeneous media is a two-dimensional $10 \mathrm{~mm} \times 10 \mathrm{~mm}$ square. The simulation geometry of this problem is shown in Fig. 4. From now on we will consider $x=x_{1}$ and $y=x_{2}$. A single isotropic light source with spatial size of $2 \mathrm{~mm}$ is symmetrically localized on the left boundary near $y=5 \mathrm{~mm}$, at $x=0 \mathrm{~mm}$, and $4 \mathrm{~mm}<y<6 \mathrm{~mm}$. Vacuum boundary conditions are applied on all the other boundaries.

This small model with area of $100 \mathrm{~mm}^{2}$ enables us to analyze the accuracy of the $P_{L}$ solutions in problems with small tissue geometries, where boundary 


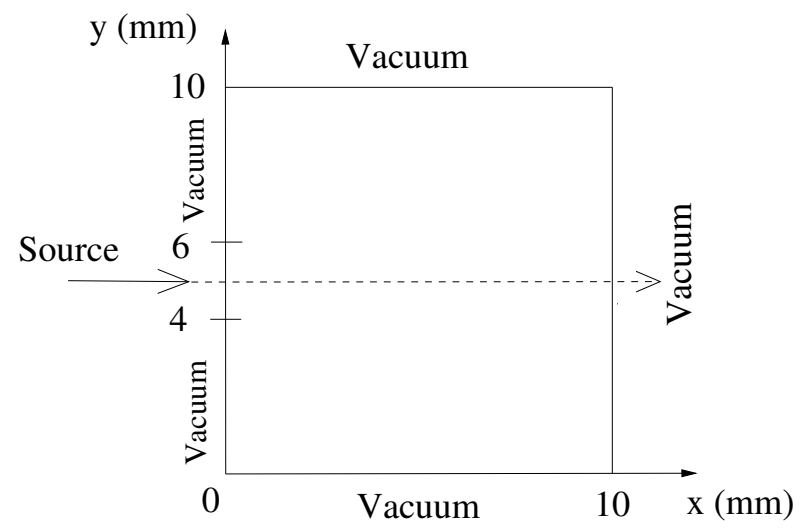

Figure 4: Geometry of the two-dimensional homogeneous medium.

effects would dominate the light propagation. These cases simulate the scattering and absorption physics of a 2D transverse tissue slice of small animals $[16]$.

We consider three numerical examples with the same geometry (see Fig. 4) by varying the absorption coefficient, the scattering coefficient, and the anisotropy factor. In the first test, we will consider a model with $\mu_{s}=0$, then we will expect large deviations between $P_{1}$ and $P_{L}$ (for $L>1$ ) solutions. Also, we will study the influence of the optical parameters on the accuracy of the $P_{L}$ solutions in media with a difussive regime where $\mu_{a} \ll \mu_{s}^{\prime}$, where a good match between all the $P_{L}$ solutions is expected, and also we will analyze the impact of the anisotropic scattering in these types of media.

The $P_{L}$ calculations were performed using a mesh consisting of $10 \times 10$ nodes with side length $1 \mathrm{~mm}$ and the order of the Legendre polynomials considered in (24) is $M=4$. We observe that in all the problems, the convergence of the nodal collocation method is already achieved when a moderate number of polynomials is considered.

In each case, the $P_{L}$ results are compared with the solution obtained with the $S_{N}$ code TWODANT [32] taken as reference, with a $500 \times 500$ grid and a convergence criterion of $10^{-7}$.

\subsubsection{D homogeneous test case $A$}

In this case the absorption coefficient considered is $\mu_{a}=0.1 \mathrm{~mm}^{-1}$ and there is no scattering. We present the $P_{L}$ photon flux solutions for odd $L$ and $1 \leq L \leq 9$. As $\mu_{s}=0$, we anticipate discrepancies between $P_{1}$ and $P_{L}(L>1)$ solutions. We also calculate the $S_{N}$ photon flux with TWODANT code and, in order to minimize the well known ray effect, we have set $N=24\left(S_{24}\right)$ in the calculations. In low scattering regions or strong absorbers the discretization schemes based on the discrete ordinates solution often exhibit rays along the discrete directions when solving the RTE [34]. The solution is highly oscillatory, 
and this effect is exacerbated by singular type sources, such as spatially localized sources. Ray effects can cause large errors in pointwise quantities such as the photon flux. The $P_{L}$ approximation does not suffer from the ray effect.

In this test case the $P_{L}$ results will be closer to the $S_{24}$ transport solution as the order $L$ increases, although one cannot draw conclusions about the accuracy of the $P_{L}$ results in comparison with the $S_{24}$ solution due to the presence of large ray effects in this latter.

The photon flux results are plotted in Fig. 5 along the horizontal line $y=5$ $\mathrm{mm}$ passing through the source. The figure shows the $P_{L}(L=1, \ldots, 9)$ and $S_{24}$ solutions.

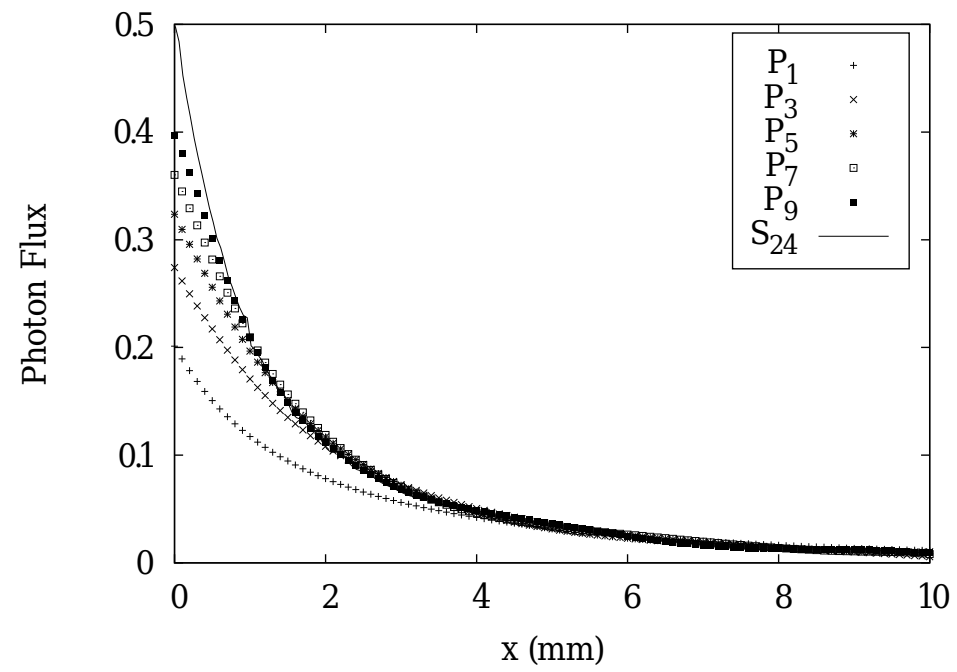

Figure 5: Photon fluxes for the homogeneous 2D case A along $y=5 \mathrm{~mm}$.

As it was expected, large deviations between $P_{1}$ and $P_{L}$ (for $L>1$ ) can be observed in Fig. 5, and the $P_{L}$ photon flux increases for large values of $L$. Along $y=5 \mathrm{~mm}$, the $P_{9}$ flux does not reach the $S_{24}$ solution. To illustrate the analysis of these results, the contour plots of the $P_{9}$ and $S_{24}$ flux distributions are given in Fig. 6, where we can see clearly that the $S_{24}$ contour map shows rays emanating from the source.

In the following sections, some tests will be treated to further analyze the accuracy of the $P_{L}$ solutions.

\subsubsection{D isotropic scattering test case $B$}

This test case is based on a work presented in [16]. We consider an isotropically scattering tissue-like medium with $\mu_{s}=1 \mathrm{~mm}^{-1}$ and $\mu_{a}=0.1 \mathrm{~mm}^{-1}$ $\left(\Rightarrow \mu_{t}=1.1 \mathrm{~mm}^{-1}\right)$, then it holds that $\mu_{a} \ll \mu_{s}$ which means that good agreement between $P_{1}, P_{L}(L>1)$ and $S_{24}$ solutions is expected. 

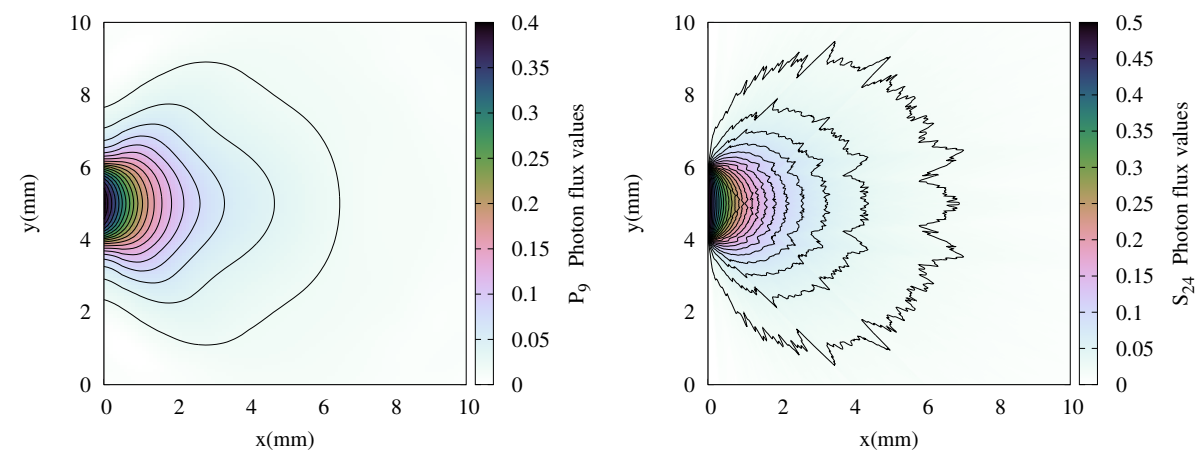

Figure 6: Contour map of the photon flux distribution, obtained with $P_{9}$ approximation (left) and $S_{24}$ (right), for the homogeneous $2 \mathrm{D}$ case A.

Fig. 7 shows the photon fluxes along $X$-axis through medium center $(y=5$ $\mathrm{mm})$, modeled by $P_{L}(L=1,3,7)$ together with the $S_{24}$ solution. The figure includes a detail of the zone for $0.8<x<1.2 \mathrm{~mm}$, where we observe that the $S_{24}$ solution presents very slight oscillations due to the ray effect.

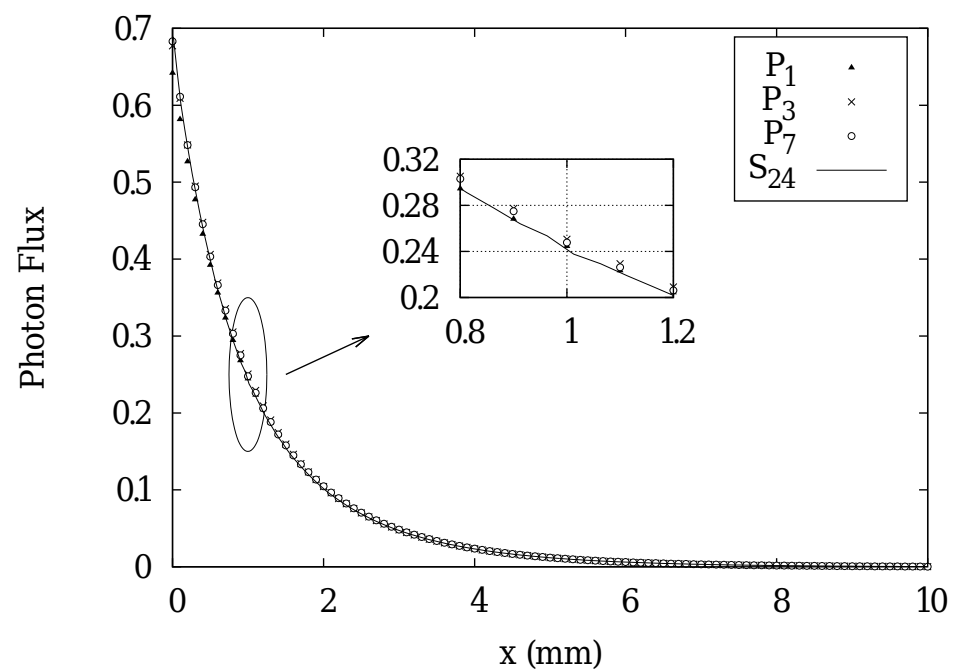

Figure 7: Photon fluxes for the homogeneous 2D case B along $y=5 \mathrm{~mm}$.

In order to quantify the difference of each $P_{L}$ photon flux with respect to the $S_{24}$ solution, we use the model error of the flux inside the medium at an interior grid point $x_{i}$ along the $X$-axis [16]. The model error $\sigma_{M i}^{\phi}$ is given by the relative percentage difference at the point $x_{i}$ of the flux $\phi_{i}$ of the $P_{L}$ approximation with respect to the flux $\widetilde{\phi}_{i}$ of the $S_{24}$ method. We also define the total model error 
$\sigma_{M}^{\phi}$ of the flux at $N$ interior grid points as the root-mean-square percentage deviation error:

$$
\sigma_{M i}^{\phi}=\frac{\phi_{i}-\widetilde{\phi}_{i}}{\widetilde{\phi}_{i}} \times 100 \% ; \quad \sigma_{M}^{\phi}=\sqrt{\frac{1}{N} \sum_{i}^{N}\left(\frac{\phi_{i}-\widetilde{\phi}_{i}}{\widetilde{\phi}_{i}}\right)^{2}} \times 100 \% .
$$

The model error of each $P_{L}$ solution, for $L=1,3,5,7$, with respect to $S_{24}$ result for this problem along the line $y=5 \mathrm{~mm}$ is shown in Fig. 8. The ray effect causes the model error oscillations, which are larger near the source. The total model errors of the flux with respect to $S_{24}$ transport solution are $\sigma_{M}^{\phi}\left(P_{1}\right)=4.32 \%$ and $\sigma_{M}^{\phi}\left(P_{7}\right)=1.43 \%$, for the $P_{1}$ and $P_{7}$ approximations respectively. The $P_{1}$ model error is higher than the $P_{7}$ model error, then the $P_{7}$ approximation performs better that the diffusion $\left(P_{1}\right)$ approximation.

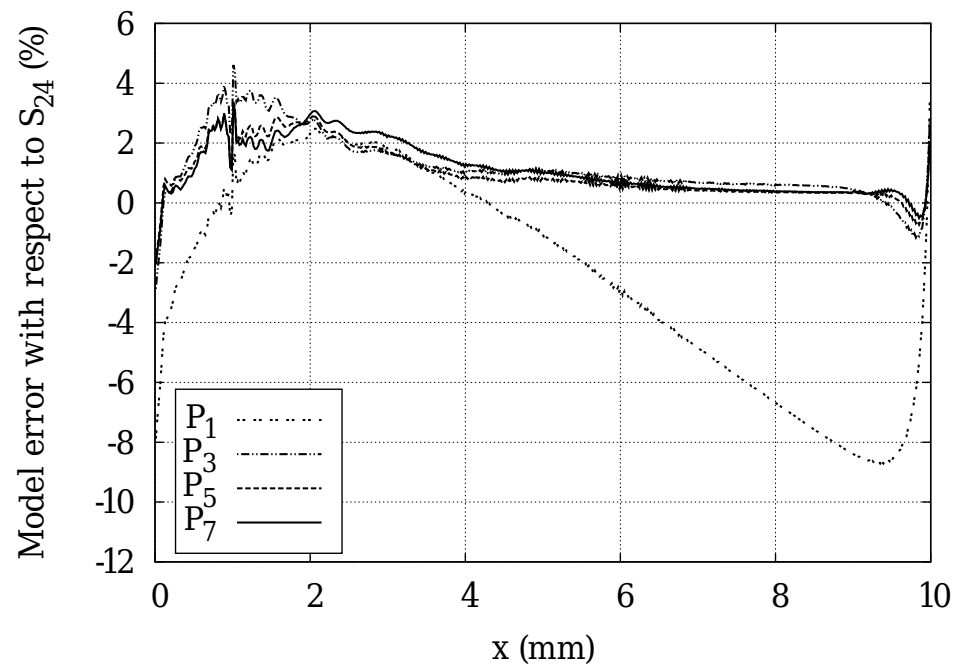

Figure 8: Relative differences of $P_{L}$ photon fluxes with respect to $S_{24}$ solution through medium center $(y=5 \mathrm{~mm})$, for $2 \mathrm{D}$ case $\mathrm{B}$.

In Fig. 9 contour maps of photon fluxes calculated with $P_{1}$ and $P_{9}$ approximations are shown in logarithmic scale. The $S_{24} \log _{10}$ flux distribution is shown in Fig. 10. Although $P_{1}$ approximation does not behave so badly in this problem, the results confirm that it is necessary to use high order $P_{L}$ approximations to obtain accurate solutions.

\subsection{3. $2 D$ anisotropic scattering test case $C$}

In this case, we define an anisotropically scattering medium whose optical properties are $\mu_{s}=5 \mathrm{~mm}^{-1}$ and $\mu_{a}=0.001 \mathrm{~mm}^{-1}$. The anisotropy factor is $g=0.8$ and the reduced scattering coefficient is $\mu_{s}^{\prime}=(1-g) \mu_{s}=1 \mathrm{~mm}^{-1}$, 

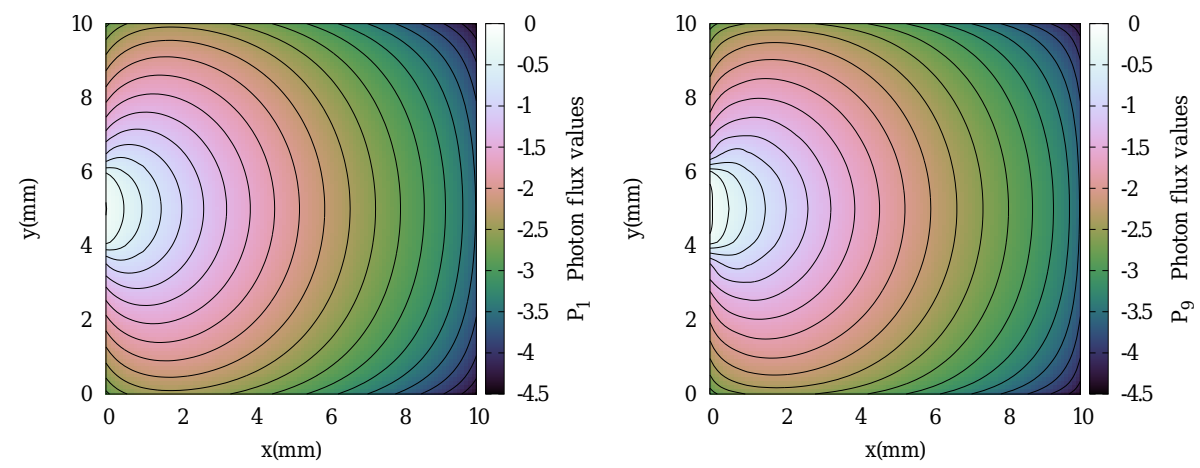

Figure 9: $\log _{10}$ of the photon flux distributions, obtained with $P_{1}$ (left), and $P_{9}$ (right) approximations, for $2 \mathrm{D}$ case $\mathrm{B}$.

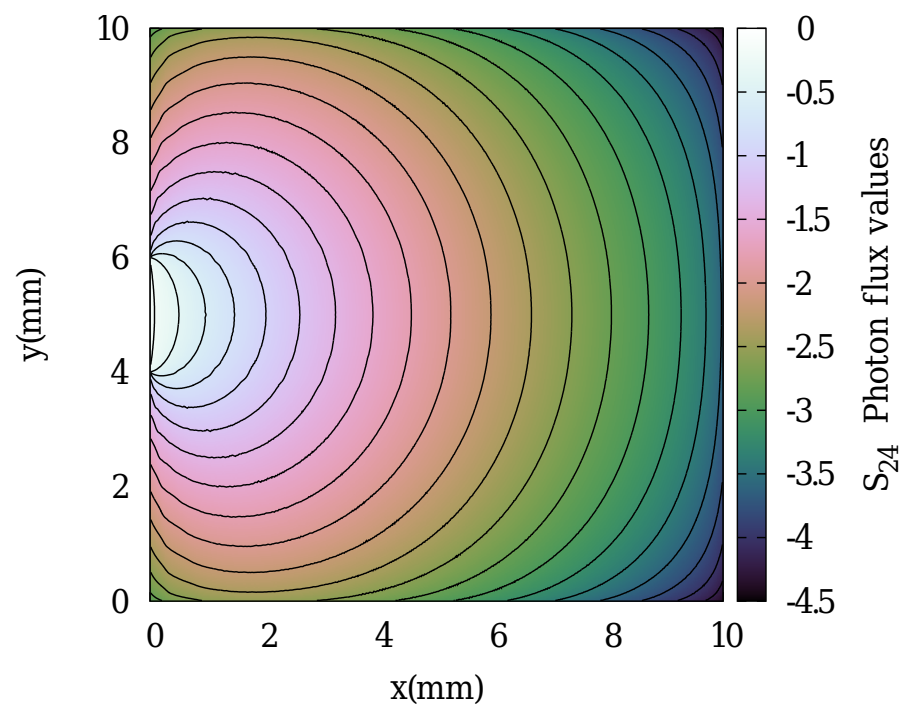

Figure 10: Contour map of the $S_{24}\left(\log _{10}\right)$ photon flux for 2 D case B.

thus we have that $\mu_{a} \ll \mu_{s}^{\prime}$. This problem is also inspired in a case presented in [16]. As $g \leq 0.8$, the scattering cross-section has been modelled according to the Henyey-Greenstein phase function (4).

The results for the photon fluxes are displayed in Figs. 11 and 13. Fig. 11 shows the photon fluxes along $X$-axis through medium center $(y=5 \mathrm{~mm})$, modeled by $P_{L}(L=1,3,5,7)$ together with the $S_{24}$ solution.

The model error of $P_{L}$ photon fluxes, for $L=1,3,5,7$, with respect to the $S_{24}$ transport solution is shown in Fig. 12.

The total model error of the flux with respect to $S_{24}$ transport solution 


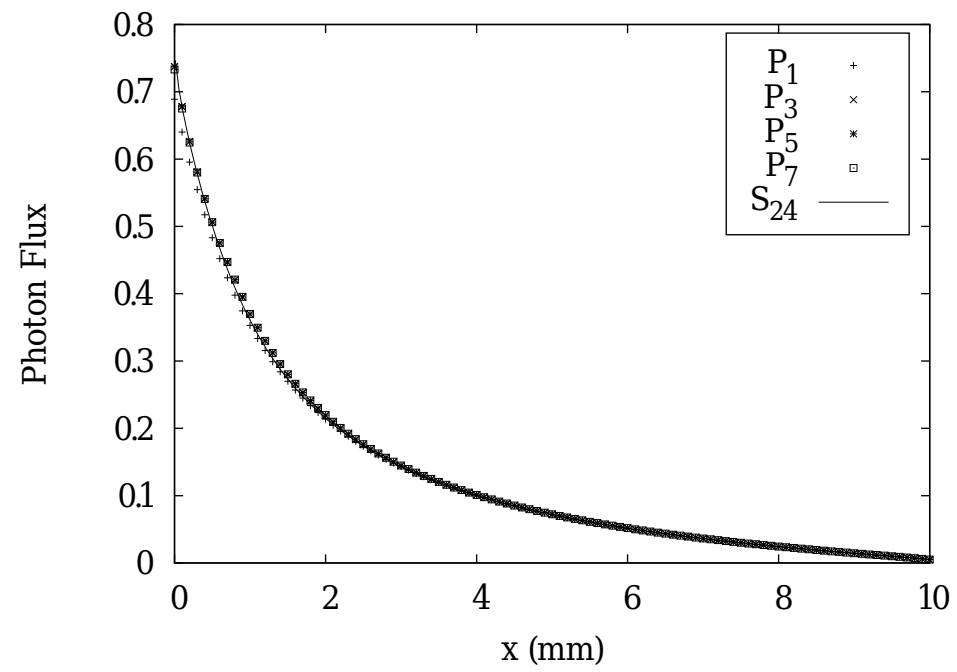

Figure 11: Photon fluxes for the anisotropic scattering case C, along the line $y=5 \mathrm{~mm}$.

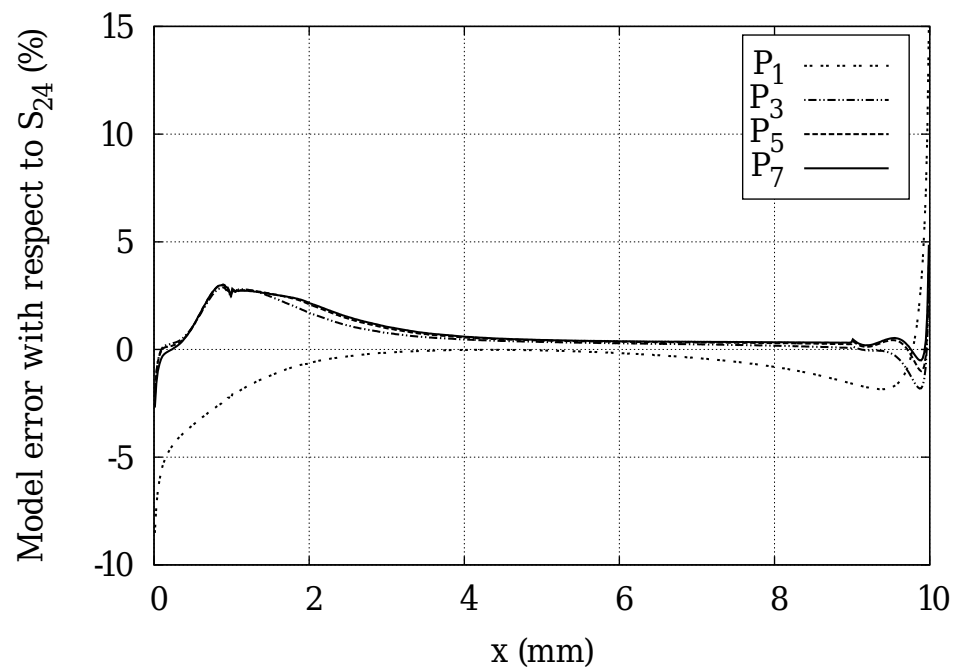

Figure 12: Relative differences of $P_{L}$ photon fluxes with respect to $S_{24}$ solution through medium center $(y=5 \mathrm{~mm})$, for $2 \mathrm{D}$ case $\mathrm{C}$.

for the $P_{1}$ approximation is $\sigma_{M}^{\phi}\left(P_{1}\right)=1.76 \%$, and for the $P_{7}$ approximation is $\sigma_{M}^{\phi}\left(P_{7}\right)=1.20 \%$. The model error $\sigma_{M}^{\phi}\left(P_{1}\right)$ is very low in this simulation, whereas it was higher in the isotropic scattering case of Section 3.2.2. Although 
in this case we have considered an anisotropic scattering medium, it is also a more diffusive problem than the isotropic case of Section 3.2.2, because now the ratio between $\mu_{a}$ and $\mu_{s}^{\prime}$ is lower $\left(\mu_{a} / \mu_{s}^{\prime}=0.001\right)$.

Fig. 13 shows the contour maps of $\log 10$ photon fluxes calculated with $P_{1}$ (left) and $P_{9}$ (right) approximations.
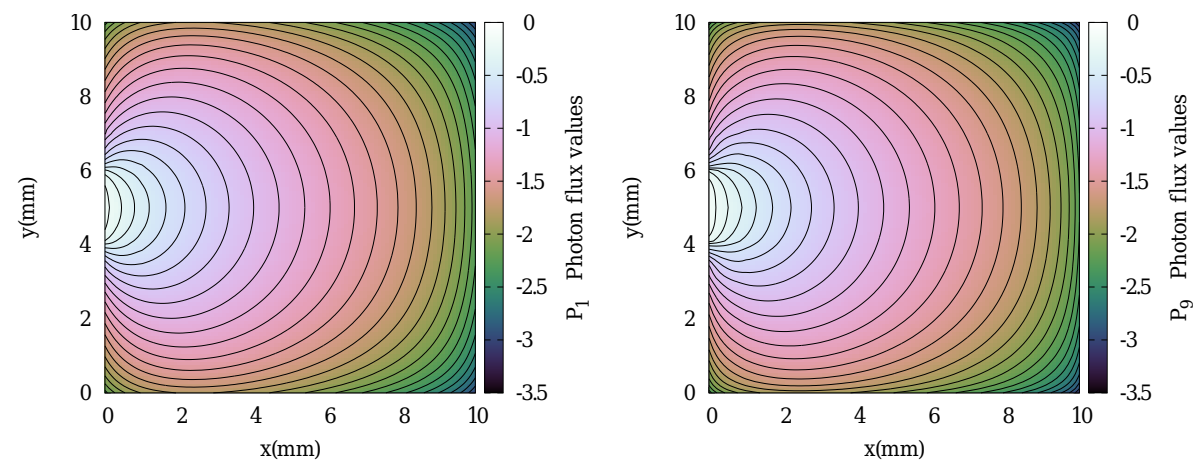

Figure 13: Contoured $\log _{10}$ of the $P_{1}$ (left) and $P_{9}$ (right) photon flux distributions, for the anisotropic scattering case $\mathrm{C}$.

The results confirm that the $P_{L}$ approximations are able to reproduce the anisotropic scattering effects on the medium.

\subsection{Two-dimensional heterogeneous media}

We study the application of the spherical harmonics-nodal collocation method in heterogeneous media whose geometry is a two-dimensional square. The first two cases considered in this Section were presented in [19] and [9], and the geometrical simulations consist of a square with an inner central squared void and a square including a channel at the periphery. The third case is chosen to analyze the effect of anisotropic scattering in heterogeneous media; in this latter case we consider the geometry of the channeling problem. In all the heterogeneous cases the value of photon flux at the external source is taken to be $10^{4}$ photons $/ \mathrm{mm}^{2}$ [35]. The numerical results show the influence of void-like regions on the transport of photons.

\subsubsection{Simulation geometry for void-like spaces}

A square region with dimensions $120 \mathrm{~mm} \times 120 \mathrm{~mm}$ enclosing smaller square region of $40 \mathrm{~mm} \times 40 \mathrm{~mm}$ is considered [19]. The geometry is shown in Fig. 14 .

We study the propagation of photons through void-like regions. Human tissue usually consists of several layers having different absorption and scattering properties. In this case, the small square region is assigned low-scattering and absorption coefficients, in order to simulate void-like regions in the human body, such as the scattering and absorption free regions in the brain, which are filled with the cerebrospinal-fluid (CSF). 


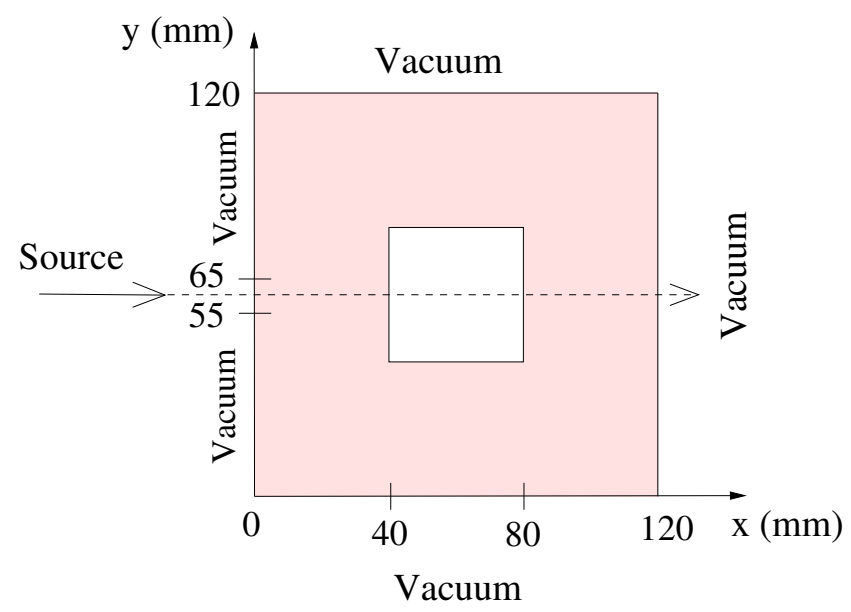

Figure 14: Geometry of two-dimensional void-like spaces.

The scattering and absorption coefficients for the outer square region are $\mu_{s}=0.5 \mathrm{~mm}^{-1}$ and $\mu_{a}=0.005 \mathrm{~mm}^{-1}$, and for the inner square $\mu_{s}=0.01$ $\mathrm{mm}^{-1}$ and $\mu_{a}=0.0001 \mathrm{~mm}^{-1}$, then $\mu_{a} \ll \mu_{s}$. An isotropic source with size 10 $\mathrm{mm}$ is symmetrically placed at $x=0 \mathrm{~mm}, 55 \mathrm{~mm}<y<65 \mathrm{~mm}$, and vacuum boundary conditions are applied on the other boundaries.

We study the accuracy of the $P_{L}$ SHNC solutions to investigate the flux distribution inside the inner square. For the $P_{L}$ calculations, a spatial discretization of the medium consisting of $24 \times 24$ square nodes with side length 5 $\mathrm{mm}$ and an order $M=4$ of Legendre polynomials has been used in the nodal collocation method. Fig. 15 shows the $\log _{10}$ of the photon flux results along the horizontal line $y=60 \mathrm{~mm}$ passing through the center of the inner square. The figure shows a comparison between the $P_{1}, P_{7}$ and $S_{24}$ solutions. The $S_{24}$ solution was calculated with a $360 \times 360$ mesh grid and a convergence criterion of $10^{-7}$.

As can be seen in Fig. 15, $P_{1}$ approximation predicts an almost constant photon flux throughout the void, whereas the $P_{7}$ and $S_{24}$ calculations show a slight attenuation of the flux inside the void. This confirms the results of Aydin et al. [19]. The total model error of the $P_{1}$ flux with respect to $S_{24}$ transport solution is $\sigma_{M}^{\phi}\left(P_{1}\right)=4.45 \%$. From the figure, we observe no significant differences between the $P_{7}$ and $S_{24}$ solutions, being the total model error of the $P_{7}$ flux $\sigma_{M}^{\phi}\left(P_{7}\right)=0.56 \%$, that confirms the capability of the high order $P_{L}$ approximation to deal with this type of problems.

In Fig. 16 contour plots of the log 10 photon fluxes are shown for the voidlike region $\left(40 \mathrm{~mm}<x<80 \mathrm{~mm}\right.$ ). The differences observed between $P_{1}$ and $P_{7}$ contour maps match those of Fig. 15. 


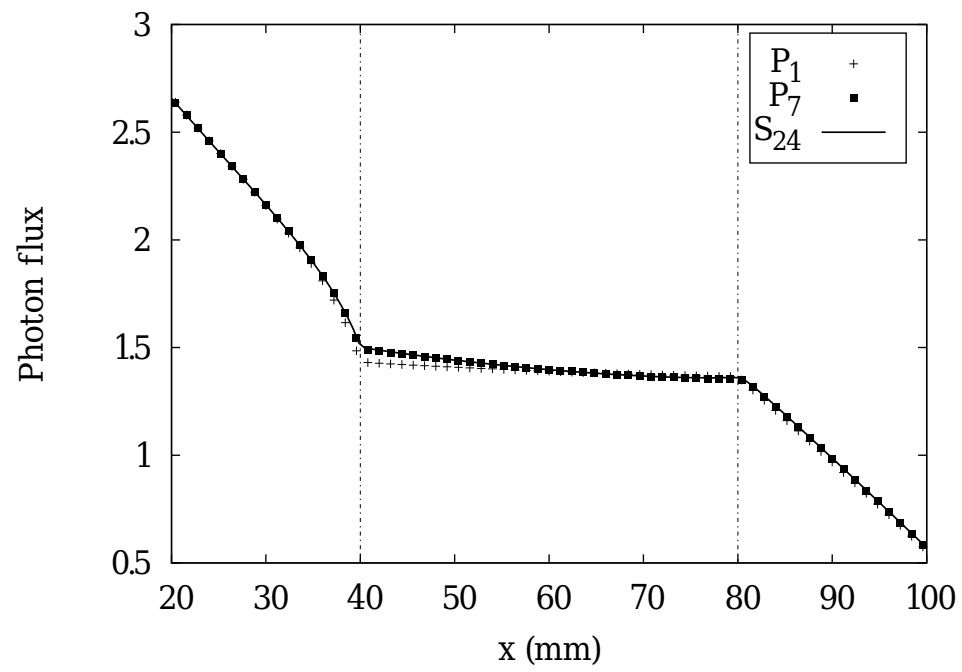

Figure 15: $\log _{10}$ of the photon flux calculated with $P_{1}, P_{7}$ and $S_{24}$, inside a low density square region.
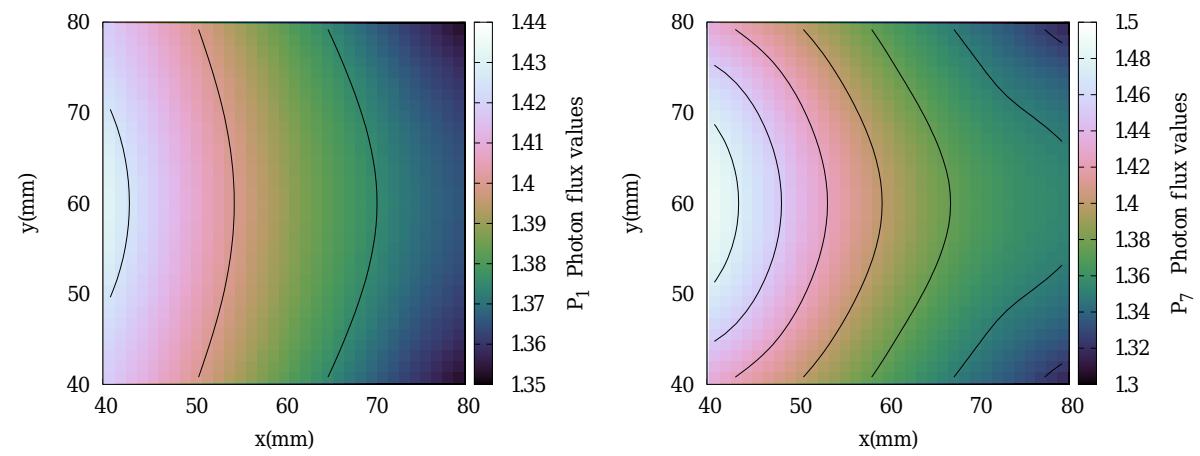

Figure 16: Contoured $\log _{10}$ of the photon flux values, obtained with $P_{1}$ (left), and $P_{7}$ (right) approximations, for the void-like region.

\subsubsection{Simulation geometry for channeling problem}

The brain is embedded in such an almost absorption and scattering-free fluid, this layer of CSF forms a boundary between the outside tissues and the brain $[9,19]$. The geometry of this problem that simulates approximately this situation is shown in Fig. 17. A $100 \mathrm{~mm} \times 100 \mathrm{~mm}$ square region with $\mu_{s}=0.5$ $\mathrm{mm}^{-1}$ and $\mu_{a}=0.005 \mathrm{~mm}^{-1}$ is considered $\left(\mu_{a} \ll \mu_{s}\right)$. The void-like region (CSF fluid) is represented by a structure of size $4 \mathrm{~mm}$ thick with $\mu_{s}=0.01$ $\mathrm{mm}^{-1}$ and $\mu_{a}=0.0001 \mathrm{~mm}^{-1}$, which surrounds a $76 \mathrm{~mm} \times 76 \mathrm{~mm}$ inner square. An isotropic source with size $4 \mathrm{~mm}$ is centered at $x=0 \mathrm{~mm}, 48 \mathrm{~mm}<y<52$ 
$\mathrm{mm}$, and vacuum boundary conditions are applied on all the other boundaries.

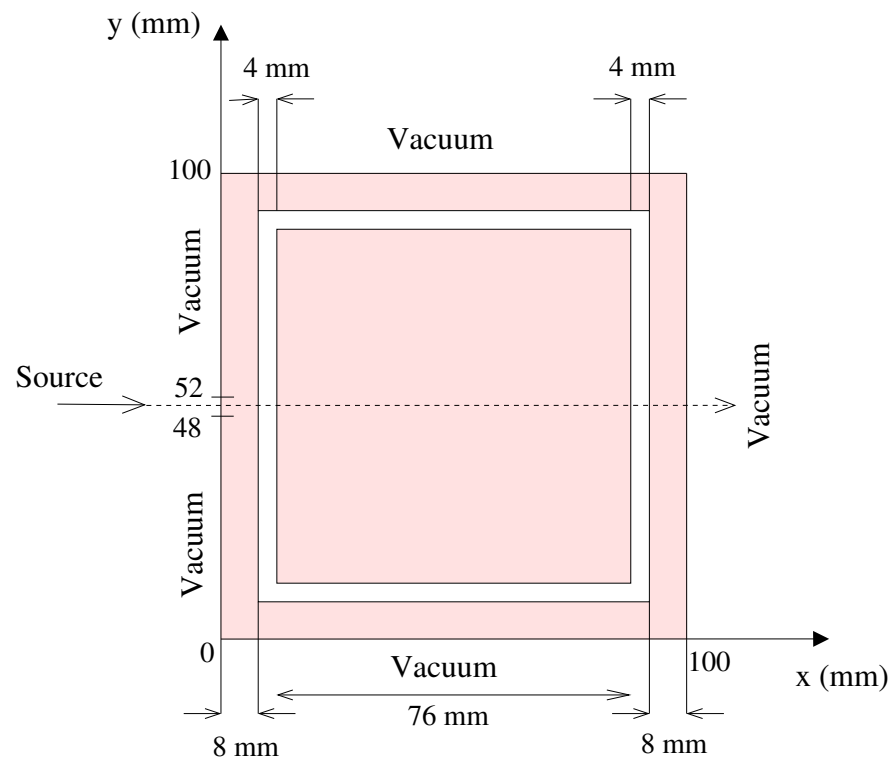

Figure 17: Geometry of two-dimensional channeling problem.

The $P_{1}, P_{7}$ and $S_{24}$ photon flux results are displayed in Fig. 18, in logarithmic scale, along the line $y=50 \mathrm{~mm}$ passing through the medium center. We consider a spatial mesh of $25 \times 25$ square nodes with side length $4 \mathrm{~mm}$ in the $P_{L}$ calculations with an order $M=4$ of Legendre polynomials, and a $1250 \times 1250$ mesh grid for the $S_{24}$ calculation, with a convergence criterion of $10^{-7}$.

Fig. 18 shows good agreement between the $P_{7}$ and $S_{24}$ solutions, being the total model error of the $P_{7}$ flux with respect to the $S_{24}$ solution $\sigma_{M}^{\phi}\left(P_{7}\right)=$ $5.97 \%$. On the other hand, the $P_{1}$ approximation shows significant discrepancies. Through the first $(8 \mathrm{~mm}<x<12 \mathrm{~mm})$ and the second $(88 \mathrm{~mm}<x<92$ $\mathrm{mm})$ void-like channels, $P_{1}$ and $P_{7}$ approximations predict an almost constant photon intensity, but inside the first channel the $P_{1}$ approximation underestimates the flux, and through the second channel $P_{1}$ flux values are higher than the ones predicted by $P_{7}$ approximation. We also observe that after the first void-like channel, the $P_{7}$ flux values show faster decay $(x>12 \mathrm{~mm})$. Unlike the $P_{1}$ results, the $P_{7}$ flux continues decreasing even behind the second channel.

In Fig. 19 contour plots of photon fluxes obtained with $P_{1}$ (left) and $P_{7}$ (right) approximations are displayed. The differences observed between $P_{1}$ and $P_{7}$ predictions in this figure just reflect the effects of the channel seen in Fig. 18. Our results also confirm the necessity of using high order $L$, in order to obtain accurate $P_{L}$ solutions of the photon fluxes especially in void-like regions. 


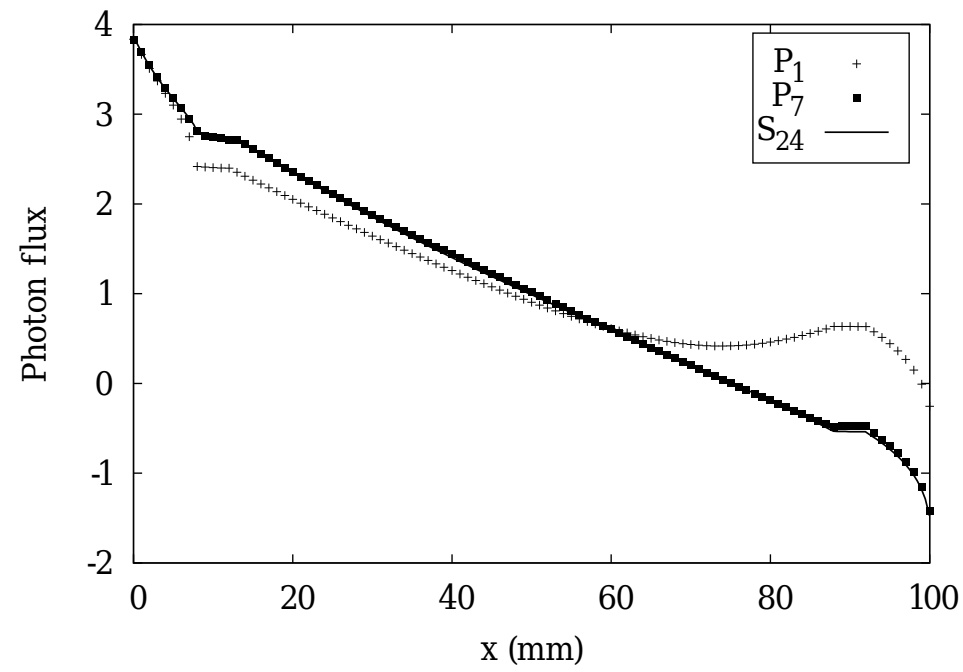

Figure 18: $\log _{10}$ of the photon fluxes for the 2D CSF-layered problem.
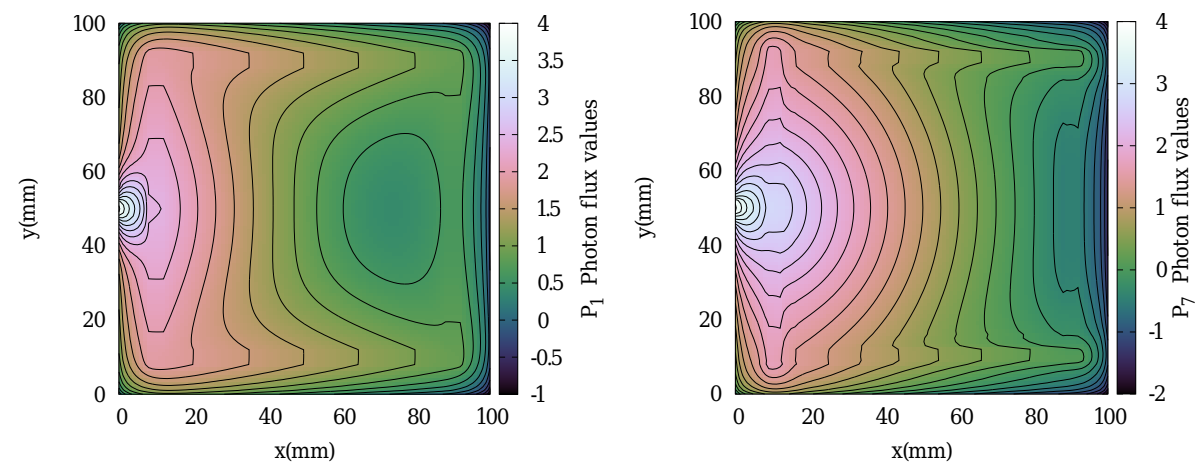

Figure 19: Contoured $\log _{10}$ of the photon flux values, obtained with $P_{1}$ (left), and $P_{7}$ (right) approximations, for the CSF-layered problem.

\subsubsection{Influence of anisotropic scattering in heterogeneous media}

As a final example we consider again the CSF-layered problem whose geometry is shown in Fig. 17. We investigate the effect of the anisotropy factor $g$ in modeling the propagation of photons through the same heterogeneous channeling problem. We take the same scattering and absorption optical properties as in Section 3.3.2 but, in this case, the anisotropy factor $g$ is taken to be 0.92 in the region outside the void-like channel and in the $76 \mathrm{~mm} \times 76 \mathrm{~mm}$ inner square, so the reduced scattering coefficient is $\mu_{s}^{\prime}=(1-g) \mu_{s}=0.04 \mathrm{~mm}^{-1}$, and $\mu_{a} / \mu_{s}^{\prime}=0.0125$ in this region. 
Fig. 20(a) shows $\log _{10}$ photon fluxes from $P_{L}(L=1,3,5,7)$ approximations along the line $y=50 \mathrm{~mm}$. For the media having anisotropy factor of $g=0.92$, we modeled the anisotropic scattering using the Henyey-Greenstein $(\mathrm{H}-\mathrm{G})$ phase function (4) with 7 Legendre expansion coefficients. We consider a spatial mesh of $25 \times 25$ nodes with side length $4 \mathrm{~mm}$.

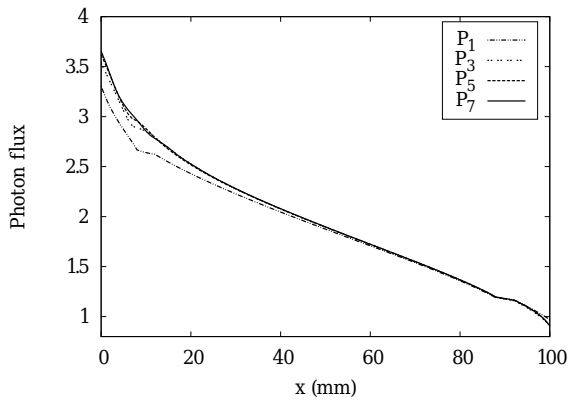

(a)

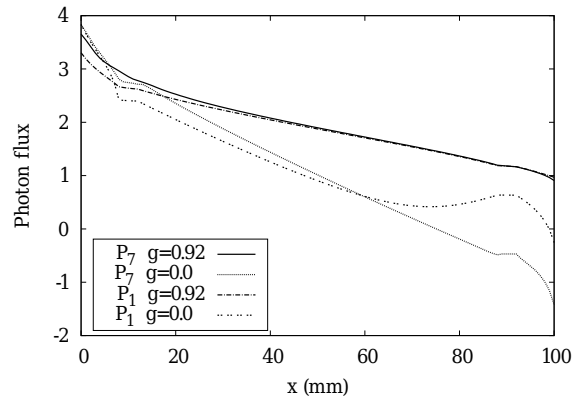

(b)

Figure 20: Influence of anisotropic scattering in heterogeneous media. In (a), $P_{1}-P_{7}$ photon fluxes. In (b), $g=0$ and $g=0.92 P_{1}$ and $P_{7}$ calculations for the same CSF-layered problem.

In Fig. 20(b) we compare the $P_{1}$ and $P_{7}$ solutions of the RTE for the case with anisotropic scattering to the corresponding solutions taking $g=0$ in both regions. Then, in this latter case we have that $\mu_{s}^{\prime}=\mu_{s}=0.5 \mathrm{~mm}^{-1}$ and $\mu_{a} / \mu_{s}^{\prime}=$ 0.01 in the region outside the channel. We observe significant differences between isotropic and anisotropic scattering cases, and $P_{1}$ and $P_{7}$ differences are more remarkable when isotropic scattering $(g=0)$ in all the media is considered. Fig. 21 displays the contoured map of the $P_{7}\left(\log _{10}\right)$ photon flux, for the same anisotropic scattering problem.

Next we present calculations for this case using the simplified approximate Mie (SAM) phase function (5) to model the anisotropic scattering. The $P_{1}$ and $P_{7} \log _{10}$ photon flux distributions calculated using the two phase functions, H-G and SAM, are compared in Fig. 22 along the horizontal line $y=50 \mathrm{~mm}$, for the left half of the medium $(0 \mathrm{~mm}<x<50 \mathrm{~mm})$. The location of the first channel is marked in the figure.

For the $P_{7}$ approximation, we used $\mathrm{H}-\mathrm{G}$ function with 7 Legendre expansion coefficients whereas only 3 Legendre coefficients were taken when the SAM function was employed. Due to the high value of the anisotropy factor $(g>0.8)$, a high number of Legendre coefficients was required for the H-G function to accurately compute the $P_{7}$ approximation. On the contrary, we numerically observe that, when dealing with SAM function, only the first 3 coefficients are required to match the accuracy of the $\mathrm{H}-\mathrm{G} P_{7}$ approximation.

From Fig. 22 we observe that the influence of the anisotropy is more important with high order $P_{L}$ approximation, and the largest discrepancies between H-G and SAM functions occur for $P_{7}$ approximation around the void-like chan- 


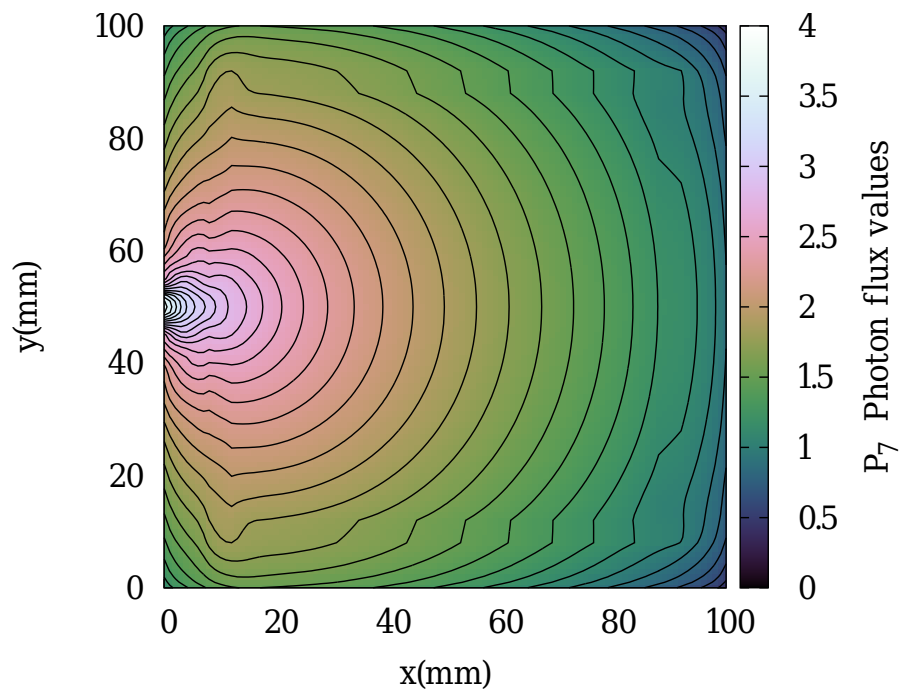

Figure 21: Contoured $\log _{10}$ of the $P_{7}$ photon flux, for the anisotropic CSF-layered problem.

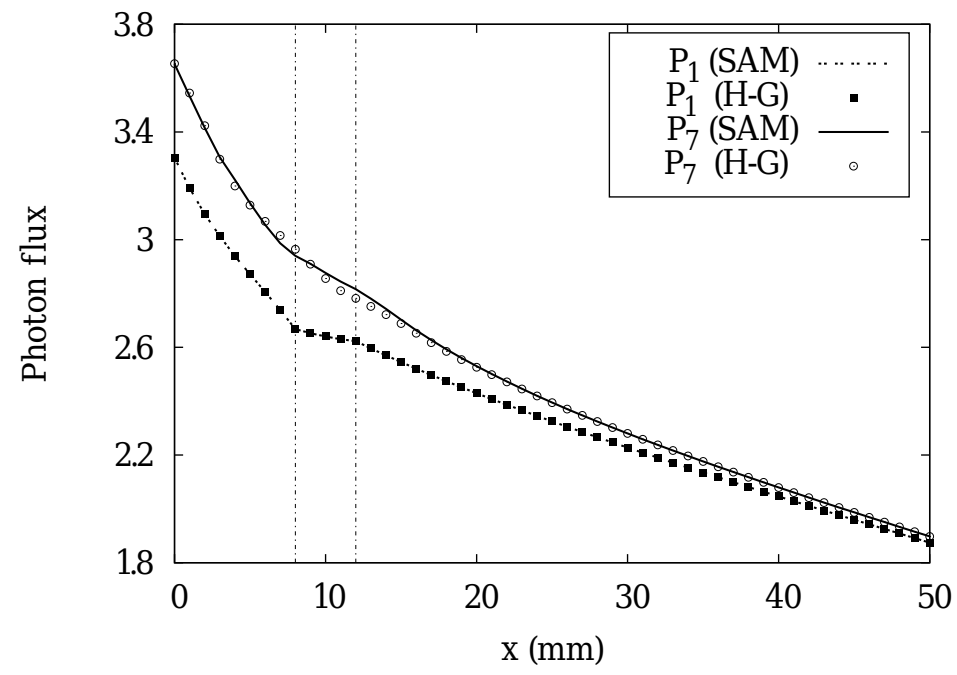

Figure 22: A comparison between the results obtained with H-G and SAM phase functions.

nel. 


\section{Conclusions}

We have reviewed the multi-dimensional spherical harmonics $P_{L}$ equations when applied to optical tomography. The $P_{L}$ equations, for arbitrary angular order $L$, have been formulated as a vector-valued diffusive second order differential equation. Surface source and vacuum boundary conditions have been approximated using Marshak's conditions and computed for arbitrary order $L$.

We have applied a nodal collocation method for the spatial discretization of the $P_{L}$ equations, based on the expansion of the angular neutronic moments in terms of orthonormal Legendre polynomials. The main advantage of the method is the lower dimension and good characteristics of the matrix associated to the algebraic problem. The method is able to work with nodes of big size using a moderate number of Legendre polynomials giving satisfactory results. The computation using big size nodes reduces the dimension of the algebraic problem in comparison to other methods like finite elements or finite differences.

As a first test of the method, we have examined the transport of photons through 1D homogeneous media, comparing the results against the exact analytical solution and also with the results provided by ONEDANT $\left(S_{N}\right)$ code, showing that $P_{1}$ approximation fails to describe the light propagation correctly, whereas higher order $P_{L}$ approximations $\left(P_{7}, P_{9}\right)$ show accurate results in comparison to the exact solution.

We have also tested several 2D homogeneous isotropic and anisotropic cases. Again, the $P_{L}$ computations are compared with the transport approximation obtained from the $S_{N}$ code TWODANT. The results confirm that the $P_{L}$ approximation is able to reproduce the isotropic and anisotropic scattering effects on the medium without suffering from ray effect as $S_{N}$ approximation does. Finally, we have studied 2D heterogeneous media with void-like regions such as low-scattering and absorption channels. In both types of media, an isotropic scattering model is studied, and in the latter case, which corresponds to the channeling problem, we consider also the anisotropic model, comparing results from the Henyey-Greenstein (H-G) and simplified approximated Mie (SAM) phase functions. The largest discrepancies between H-G and SAM phase functions occur for $P_{7}$ approximation around the void-like channel. We remark the necessity of using $P_{L}$ approximations for $L>1$, even in cases where the diffusion approximation does not fail completely. Due to the ill-posed nature of these type of problems, even small errors in modeling can cause large errors in the reconstructions. Therefore, it is important to have a computationally feasible model that describes light propagation in the medium accurately.

In summary, in this work we have shown that the diffusive $P_{L}$ spherical harmonics equations have several advantages if they are compared with $S_{N}$ or $S P_{L}$ equations and that this model can be efficiently applied to optical tomography obtaining good results. 


\section{Acknowledgments}

This work has been partially supported by the Spanish Ministerio de Economía y Competitividad under project ENE-2014-59442-P and by the Generalitat Valenciana under project PROMETEO II/2014/008.

\section{References}

[1] Haisch C. Optical Tomography. Annu Rev Anal Chem 2012;5:55-77.

[2] Qi J, Ye Z. Computed Tomography Laser Mammography Optical as an adjunct to mammography in the diagnosis of patients with dense breast. Clin Imag 2013;37(2):289-294.

[3] Klose AD, Netz U, Beuthan J, Hielscher AH. Optical tomography using the time-independent equation of radiative transfer Part 1: forward model. J Quant Spectrosc Radiat Transf 2002;72(5):691-713.

[4] Dudko OK, Weiss GH. Photon Diffusion in Biological Tissues. Diffusion Fundamentals 2005;2:114.1-114.21.

[5] Venugopal V, Intes X. Recent advances in optical mammography. Curr Med Imaging Rev 2012;8(3):244-259.

[6] Aydin ED, De Oliveira CRE, Goddard AJH. A finite element-spherical harmonics radiation transport model for photon migration in turbid media. J Quant Spectrosc Radiat Transf 2004;84:247-260.

[7] Boas DA. Diffuse photon probes of structural and dynamical properties of turbid media: Theory and biomedical applications. PhD Thesis, University of Pennsylvania, USA, 1996.

[8] Gibson AP, Hebden JC, Arridge SR. Recent advances in diffuse optical imaging. Phys Med Biol 2005;50(4):R1-R43.

[9] Hielscher AH, Alcouffe RE, Barbour RL. Comparison of finite-difference transport and diffusion calculations for photon migration in homogeneous and heterogeneous tissues. Phys Med Biol 1998;43:1285-1302.

[10] Arridge SR, Dehghani H, Schweiger M, Okada E. The finite element model for the propagation of light in scattering media: a direct method for domains with nonscattering regions. Med Phys 2000;27:252-64.

[11] Firbank M, Arridge SR, Schweiger M, Delpy DT. An investigation of light transport through scattering bodies with non-scattering regions. Phys Med Biol 1996;41:767-83.

[12] Arridge SR. Optical tomography in medical imaging. Inverse Problems 1999;15:R41-R93. 
[13] Tahir KB, De Oliveira CRE, Katsimichas S, Dainty JC. A comparison between finite element transport and Monte Carlo solutions for photon propagation. Proc Appl Opt Opto-electronics 1996: 58-63.

[14] Kaltenbach JM, Kaschke M. Frequency- and time-domain modelling of light transport in random media. Proc. SPIE ISll, 1993. p. 65-86.

[15] Josef JA, Morel JE. Simplified spherical harmonic method for coupled electronphoton transport calculations. Phys Rev E 1998;57(5):6161-6171.

[16] Klose AD, Larsen EW. Light transport in biological tissue based on the simplified spherical harmonics equations. J Comp Phys 2006;220:441-470.

[17] Brunner TA, Holloway JP. Two dimensional time dependent Riemann solvers for neutron transport. J Comput Phys 2005;210(1):386-399.

[18] McClarren RG, Hollway JP, Brunner TA. Analytic $P_{1}$ solutions for timedependent, thermal radiative transfer in several geometries. J Quant Spectrosc Radiat Transf 2008;109:389-403.

[19] Aydin ED, De Oliveira CRE, Goddard AJH. A comparison between transport and diffusion calculations using a finite element-spherical harmonics radiation transport method. Med Phys 2002;29(9):2013-2023.

[20] Duderstadt JJ, Hamilton LJ. Nuclear Reactor Analysis. New York: Wiley, 1976.

[21] Courant R, Hilbert D. Methods of Mathematical Physics, Vol. I. WileyInterscience, 1962.

[22] Stratton A. Electromagnetic Theory. New York: McGraw-Hill, 1941.

[23] Van de Hulst HC. Multiple Light Scattering: Tables, Formulas and Applications. New York: Academic, 1980.

[24] Liu P. A new phase function approximating to Mie scattering for radiative transport equations. Phys Med Biol 1994;39:1025-1036.

[25] Stacey WM. Nuclear Reactor Physics. New York: Wiley, 2001.

[26] Hébert A. Development of the nodal collocation method for solving the neutron diffusion equation. Ann Nucl Energ 1987;14(10):527-541.

[27] Verdú G, Ginestar D, Vidal V, Muñoz-Cobo JL. $\lambda$ modes of the neutron diffusion equation. Ann Nucl Energ 1994;21(7):405-421.

[28] Capilla M, Talavera CF, Ginestar D, Verdú G. A nodal collocation method for the calculation of the lambda modes of the $P_{L}$ equations. Ann Nucl Energ 2005;32:1825-1853. 
[29] Capilla M, Talavera CF, Ginestar D, Verdú G. Application of a nodal collocation approximation for the multidimensional $P_{L}$ equations to the 3D Takeda benchmark problems. Ann Nucl Energ 2012;40:1-13.

[30] Saad Y. SPARSKIT: a basic tool kit for sparse matrix computationsVersion 2. http://www-users.cs.umn.edu/ ${ }^{\sim}$ saad/software/SPARSKIT, 1994.

[31] Capilla M, Talavera CF, Ginestar D, Verdú G. Nodal collocation method for the multidimensional $P_{L}$ equations applied to neutron transport source problems. Ann Nucl Energ 2016;87:89-100.

[32] Alcouffe RE, Baker RS, Brinkley FW, Marr DR, O'Dell RD, Walters WF. DANTSYS: A diffusion accelerated neutral particle transport code system. Los Alamos National Laboratory, LA-12969-M, 1995.

[33] Piessens R, Doncker-Kapenga E, Überhuber CW, Kahaner D. QUADPACK: A subroutine package for automatic integration. Springer-Verlag, ISBN 978-3-540-12553-2, 1983.

[34] O'Dell RD, Alcouffe RE. Transport calculations for nuclear analyses: theory and guidelines for effective use of transport codes. ANL Manuscript LA-10983-MS, 1987.

[35] Aydin ED. Three-dimensional photon migration through voidlike regions and channels. Appl Opt 2007;46(34):8272-8277.

[36] Mohan PS, Tarvainen T, Schweiger M, Pulkkinen A, Arridge SR. Variable order spherical harmonic expansion scheme for the radiative transport equation using finite elements. J Comp Phys 2011;230:7364-7383. 